\title{
Kocay's lemma, Whitney's theorem, and some polynomial invariant reconstruction problems
}

\author{
Bhalchandra D. Thatte \\ Allan Wilson Centre for Molecular Ecology and Evolution, \\ and Institute of Fundamental Sciences, \\ Massey University, Palmerston North, New Zealand \\ b. thatte@massey.ac.nz
}

Submitted: Jun 29, 2004; Accepted: Nov 7, 2005; Published: Nov 25, 2005

Mathematics Subject Classifications: 05C50, 05C60

\begin{abstract}
Given a graph $G$, an incidence matrix $\mathcal{N}(G)$ is defined on the set of distinct isomorphism types of induced subgraphs of $G$. It is proved that Ulam's conjecture is true if and only if the $\mathcal{N}$-matrix is a complete graph invariant. Several invariants of a graph are then shown to be reconstructible from its $\mathcal{N}$-matrix. The invariants include the characteristic polynomial, the rank polynomial, the number of spanning trees and the number of hamiltonian cycles in a graph. These results are stronger than the original results of Tutte in the sense that actual subgraphs are not used. It is also proved that the characteristic polynomial of a graph with minimum degree 1 can be computed from the characteristic polynomials of all its induced proper subgraphs. The ideas in Kocay's lemma play a crucial role in most proofs. Kocay's lemma is used to prove Whitney's subgraph expansion theorem in a simple manner. The reconstructibility of the characteristic polynomial is then demonstrated as a direct consequence of Whitney's theorem as formulated here.
\end{abstract}

\section{Introduction}

Suppose we are given the collection of induced subgraphs of a graph. There is a natural partial order on this collection defined by the induced subgraph relationship between members of the collection. An incidence matrix may be constructed to represent this relationship along with the multiplicities with which members of the collection appear as induced subgraphs of other members. Given such a matrix, is it possible to construct the graph or compute some of its invariants? Such a question is motivated by the treatment of chromatic polynomials in Biggs [2]. Biggs demonstrates that it is possible to compute the chromatic polynomial of a graph from its incidence matrix. The idea of Kocay's lemma in graph reconstruction theory is extremely useful in studying the question for other 
invariants. In this paper, we present several results on a relationship between Ulam's reconstruction conjecture and the incidence matrix. Extending the reconstruction results of Tutte and Kocay, we show that many graph invariants can be computed from the incidence matrix. We then consider the problem of computing the characteristic polynomial of a graph from the characteristic polynomials of all induced proper subgraphs. Finally, we present a new short proof of Whitney's subgraph expansion theorem, and demonstrate the reconstructibility of the characteristic polynomial of a graph using Whitney's theorem.

\section{$1.1 \quad$ Notation}

We consider only finite simple graphs in this paper. Let $G$ be a graph with vertex set $V G$ and edge set $E G$. The number of vertices of $G$ is denoted by $v(G)$ and the number of edges is denoted by $e(G)$. When $V G=\emptyset$, we denote $G$ by $\Phi$, and call the graph a null graph. When $E G=\emptyset$, we call the graph an empty graph. When $F$ is a subgraph of $G$, we write $F \subseteq G$, and when $F$ is a proper subgraph of $G$, we write $F \subsetneq G$. The subgraph of $G$ induced by $S \subseteq V G$ is the subgraph whose vertex set is $S$ and whose edge set contains all the edges having both end vertices in $S$. It is denoted by $G_{S}$. The subgraph of $G$ induced by $V G-S$ is denoted by $G-S$, or simply $G-u$ if $S=\{u\}$. A subgraph of $G$ with vertex set $V \subseteq V G$ and edge set $E \subseteq E G$ is denoted by $G_{(V, E)}$, or just $G_{E}$ if $V$ consists of the end vertices of edges in $E$. The same notation is used when $E=\left(e_{1}, e_{2}, \ldots, e_{k}\right)$ is a tuple of edges, some of which may be identical. Isomorphism of two graphs $G$ and $H$ is denoted by $G \cong H$. For $i>0$, a graph isomorphic to a cycle of length $i$ is denoted by $C_{i}$, and the number of cycles of length $i$ in $G$ is denoted by $\psi_{i}(G)$, where, as a convention, $C_{i} \cong K_{i}$ for $i \in\{1,2\}$. The number of hamiltonian cycles is denoted by a special symbol $\operatorname{ham}(G)$ instead of $\psi_{v(G)}(G)$. While counting the number of subgraphs of a graph $G$ that are isomorphic to a graph $F$, it is important to make a distinction between induced subgraphs and edge subgraphs. The number of subgraphs of $G$ that are isomorphic to $F$ is denoted by $\left[\begin{array}{l}G \\ F\end{array}\right]$, and the number of induced subgraphs of $G$ that are isomorphic to $F$ is denoted by $\left(\begin{array}{c}G \\ F\end{array}\right)$. The two numbers are related by

$$
\left[\begin{array}{c}
G \\
F
\end{array}\right]=\sum_{H \mid V H=V F}\left(\begin{array}{l}
G \\
H
\end{array}\right)\left[\begin{array}{l}
H \\
F
\end{array}\right]
$$

where the summation is over distinct isomorphism types of graphs $H$. The characteristic polynomial of $G$ is denoted by $P(G ; \lambda)=\sum_{i=0}^{v(G)} c_{i}(G) \lambda^{v(G)-i}$. The collection $\mathcal{P D}(G)=$ $\{P(G-S ; \lambda) \mid S \subsetneq V G\}$ is called the complete polynomial deck of $G$. Note that a polynomial may appear in the collection more than once. The rank of a graph $G$, which has $\operatorname{comp}(G)$ components, is defined by $v(G)-\operatorname{comp}(G)$, and its co-rank is defined by $e(G)-$ $v(G)+\operatorname{comp}(G)$. The rank polynomial of $G$ is defined by $R(G ; x, y)=\sum \rho_{r s} x^{r} y^{s}$, where $\rho_{r s}$ is the number of subgraphs of $G$ with rank $r$ and co-rank $s$. The set of consecutive integers from $a$ to $b$ is denoted by $[a, b]$; in particular, $N_{k}=[1, k]$. 


\subsection{Ulam's Conjecture}

The vertex deck of a graph $G$ is the collection $\mathcal{V D}(G)=\{G-v \mid v \in V G\}$, where the subgraphs in the collection are 'unlabelled' (or isomorphism types). Note that the vertex deck is not exactly a set: an isomorphism type may appear more than once in the vertex deck. A Graph $G$ is said to be reconstructible if its isomorphism class is determined by $\mathcal{V D}(G)$. Ulam [16] proposed the following conjecture.

Conjecture 1.1. Graphs on more than 2 vertices are reconstructible.

A property or an invariant of a graph $G$ is said to be reconstructible if it can be calculated from $\mathcal{V D}(G)$. For example, Kelly's Lemma allows us to count the number of vertex-proper subgraphs of $G$ of any given type.

Lemma 1.2. (Kelly's Lemma [7]) If $F$ is a graph such that $v(F)<v(G)$ then

$$
\left[\begin{array}{c}
G \\
F
\end{array}\right]=\frac{1}{v(G)-v(F)} \sum_{u \in V G}\left[\begin{array}{c}
G-u \\
F
\end{array}\right],
$$

so $\left[\begin{array}{l}G \\ F\end{array}\right]$ is reconstructible from $\mathcal{V D}(G)$. Also, in Equation (2), $\left[\begin{array}{c}G \\ F\end{array}\right]$ and $\left[\begin{array}{c}G-u \\ F\end{array}\right]$ may be replaced by $\left(\begin{array}{c}G \\ F\end{array}\right)$ and $\left(\begin{array}{c}G-u \\ F\end{array}\right)$, respectively.

Tutte [14], [15] proved the reconstructibility of the characteristic polynomial and the chromatic polynomial. Tutte's results were simplified by an elegant counting argument by Kocay [8]. This argument is useful to count certain subgraphs that span $V G$.

Let $S=\left\{F_{1}, F_{2}, \ldots, F_{k}\right\}$ be a family of graphs. Let $c(S, H)$ be the number of tuples $\left(X_{1}, X_{2}, \ldots, X_{k}\right)$ of subgraphs of $H$ such that $X_{i} \cong F_{i} \forall i$, and $\cup_{i=1}^{k} X_{i}=H$. We call it the number of $S$-covers of $H$.

\section{Lemma 1.3. (Kocay's Lemma [8])}

$$
\prod_{i=1}^{k}\left[\begin{array}{c}
G \\
F_{i}
\end{array}\right]=\sum_{X} c(S, X)\left[\begin{array}{c}
G \\
X
\end{array}\right]
$$

where the summation is over all isomorphism types of subgraphs of $G$. Also, if $v\left(F_{i}\right)<$ $v(G) \forall i$ then $\sum_{X} c(S, X)\left[\begin{array}{l}G \\ X\end{array}\right]$ over all isomorphism types $X$ of spanning subgraphs of $G$ can be reconstructed from the vertex deck of $G$.

We refer to [3] for a survey of reconstruction problems. 


\subsection{The chromatic polynomial and the $\mathcal{N}$-matrix}

Stronger reconstruction results on the chromatic polynomial were implicit in Whitney's work [17], although Ulam's conjecture had not been posed at the time. Motivation for some of the work presented in this paper comes from Whitney's work on the chromatic polynomials. The discussion of the chromatic polynomial presented here is based on [2].

A graph $G$ is called quasi-separable if there exists $K \subsetneq V G$ such that $G_{K}$ is a complete graph and $G-K$ is disconnected. If $|K| \leq 1$ then $G$ is said to be separable.

Theorem 1.4. (Theorem 12.5 in [2]) The chromatic polynomial of a graph is determined by its proper induced subgraphs that are not quasi-separable.

The procedure of computing the chromatic polynomial may be outlined as follows. First a matrix $\mathcal{N}(G)=\left(N_{i j}\right)$ is constructed. The rows and the columns of $\mathcal{N}(G)$ are indexed by induced subgraphs $\Lambda_{1}, \Lambda_{2}, \ldots, \Lambda_{I}=G$, which are the distinct isomorphism types of non-quasi-separable induced subgraphs of $G$. The list includes $K_{1}=\Lambda_{1}$ and $K_{2}=\Lambda_{2}$. The indexing graphs are ordered in such a way that $v\left(\Lambda_{i}\right)$ are in non-decreasing order. The entry $N_{i j}$ is the number of induced subgraphs of $\Lambda_{i}$ that are isomorphic to $\Lambda_{j}$. It is a lower triangular matrix with diagonal entries 1 . The computation of the chromatic polynomial is performed by a recursive procedure beginning with the first row of the $\mathcal{N}$-matrix, computing at each step certain polynomials in terms of the corresponding polynomials for non-quasi-separable induced subgraphs on fewer vertices. A few observations about the procedure are useful to motivate the work in this paper. The graphs $C_{4}$ and $K_{4}$ are the only non-quasi-separable graphs on 4 vertices. Also, for any $i, N_{i 1}=v\left(\Lambda_{i}\right)$, and $N_{i 2}=e\left(\Lambda_{i}\right)$. Therefore, graphs on 4 or fewer vertices that index the first few rows of the $\mathcal{N}$-matrix can be inferred from the matrix entries. Therefore, we conclude that the computation of the chromatic polynomial can be performed on the matrix entries alone, even if the induced subgraphs indexing the rows and the columns of $\mathcal{N}(G)$ are unspecified. Therefore, we will think of the $\mathcal{N}$-matrix as unlabelled, that is, we will assume that the induced subgraphs indexing the rows and the columns are not given.

A natural question is what other invariants can be computed from the (unlabelled) $\mathcal{N}$-matrix? Obviously, the characteristic polynomial $P(G ; \lambda)$ cannot always be computed from $\mathcal{N}(G)$. For example, the only non-quasi-separable induced subgraphs of any tree $T$ are $K_{1}$ and $K_{2}$, so $P(T ; \lambda)$ cannot be computed from $\mathcal{N}(T)$. Therefore, we omit the restriction of non-quasi-separability on the induced subgraphs used in the construction of the incidence matrix. We then investigate which invariants of a graph are determined by its $\mathcal{N}$-matrix.

The Sections 2 and 3 are devoted to the study of reconstruction from the $\mathcal{N}$-matrix. In Section 2, we formally define the $\mathcal{N}$-matrix, and the related concept of the edge labelled poset of induced subgraphs of a graph. We then prove several basic results on the relationship between the $\mathcal{N}$-matrix, the edge labelled poset and reconstruction. In particular, we show that Ulam's conjecture is true if and only if the $\mathcal{N}$-matrix itself is a complete graph invariant. We then prove that Ulam's conjecture is true if and only if the edge labelled poset has no non-trivial automorphisms. We also prove the $\mathcal{N}$-matrix reconstructibility of trees and forests. 
In Section 3 we compute several invariants of a graph from its $\mathcal{N}$-matrix. We prove that the characteristic polynomial $P(G ; \lambda)$ of a graph $G$, its rank polynomial $R(G ; x, y)$, the number of spanning trees in $G$, the number of Hamiltonian cycles in $G$ etc., can be computed from $\mathcal{N}(G)$. In the standard proof of the reconstructibility of these invariants, one first counts the disconnected subgraphs of each type, (see [3]). In view of Theorem 2.12, the proofs in Section 3 are more involved. Theorem 2.12 implies that if there are counter examples to Ulam's conjecture then there are many more counter examples to reconstruction from the $\mathcal{N}$-matrix. Therefore, we hope that the study of $\mathcal{N}$-matrix reconstructibility will highlight new difficulties. Similar generalisations of the reconstruction problem were also suggested by Tutte, (notes on pp. 123-124 in [15]).

\subsection{Reconstruction of the characteristic polynomial}

The proof of the reconstructibility of the characteristic polynomial of a graph from its $\mathcal{N}$-matrix is also of independent technical interest, since other authors have considered the question of computing $P(G ; \lambda)$ given the polynomial deck $\{P(G-u ; \lambda) ; u \in V G\}$. This question was originally proposed by Gutman and Cvetković [5], and has been studied by others, for example, [9] \& [10]. This question remains open. So we consider a weaker question in Section 4: the question of computing the characteristic polynomial of a graph from its complete polynomial deck. Here we present basic facts about the characteristic polynomial, and outline the idea of Section 4.

Definition 1.5. A graph is called elementary if each of its components is 1-regular or 2 -regular. In other words, each component of an elementary graph is a single edge $\left(K_{2}\right)$ or a cycle $\left(C_{r} ; r>2\right)$.

Let $L_{i}$ be the collection of all unlabelled $i$-vertex elementary graphs. So, $L_{0}=\{\Phi\}$, $L_{1}=\emptyset, L_{2}=\left\{K_{2}\right\}$, and so on.

Lemma 1.6. (Proposition 7.3 in [2]) Coefficients of the characteristic polynomial of a graph $G$ are given by

$$
(-1)^{i} c_{i}(G)=\sum_{F \in L_{i}, F \subseteq G}(-1)^{r(F)} 2^{s(F)}
$$

where $r(F)$ and $s(F)$ are the rank and the co-rank of $F$, respectively.

Thus, $c_{0}(G)=1, c_{1}(G)=0$, and $c_{2}(G)=e(G)$.

Lemma 1.7. (Note 2d in [2]) Let $P^{\prime}(G ; \lambda)$ denote the first derivative of $P(G ; \lambda)$ with respect to $\lambda$. Then,

$$
P^{\prime}(G ; \lambda)=\sum_{u \in V G} P(G-u ; \lambda)
$$

From the above two lemmas, it is clear that the problem of reconstructing a characteristic polynomial (either from the vertex deck or the complete polynomial deck) reduces to computing the coefficient $c_{v(G)}(G)$, which is the constant term in $P(G ; \lambda)$. This 
in turn is a problem of counting the elementary spanning subgraphs of $G$ - a problem that can be solved using Kocay's Lemma in case of reconstruction from the vertex deck. Motivated by Kocay's Lemma, we ask the following question. Suppose the coefficients $c_{i_{1}}(G), c_{i_{1}}(G), \ldots, c_{i_{k}}(G)$ are known, and $i_{1}+i_{2}+\ldots+i_{k} \geq v(G)$. If the coefficients $c_{i_{j}} ; 1 \leq j \leq k$ are multiplied, can we get some information about the spanning subgraphs of $G$ ? This is especially tempting if $i_{1}+i_{2}+\ldots+i_{k}=v(G)$, since the product is expected to have some relationship with the disconnected spanning elementary subgraphs of $G$. This idea is explored in Section 4.

In Section 5, we present a very simple new proof of Whitney's subgraph expansion theorem, again based on Kocay's lemma. We then present a more direct argument to compute the characteristic polynomial of a graph from its vertex deck, based on our formulation of Whitney's theorem.

\section{Ulam's conjecture and the $\mathcal{N}$-matrix}

Let $\Lambda(G)=\left\{\Lambda_{i} ; i \in[1, I]\right\}$ be the set of distinct isomorphism types of nonempty induced subgraphs of $G$. We call this the $\Lambda$-deck of $G$. Let $\mathcal{N}(G)=\left(N_{i j}\right)$ be an $I$ x $I$ incidence matrix where $N_{i j}$ is the number of induced subgraphs of $\Lambda_{i}$ that are isomorphic to $\Lambda_{j}$. Thus $N_{i i}$ is 1 for all $i \in[1, I]$. We call an invariant of a graph $\mathcal{N}$-matrix reconstructible if it can be computed from the (unlabelled) $\mathcal{N}$-matrix of the graph.

As an example, the ladder graph $L_{3}$ and its collection of distinct induced subgraphs with nonempty edge sets are shown in Figure 1 . Below each graph (except $L_{3}$ ) is shown its multiplicity as an induced subgraph in $L_{3}$ and its name.
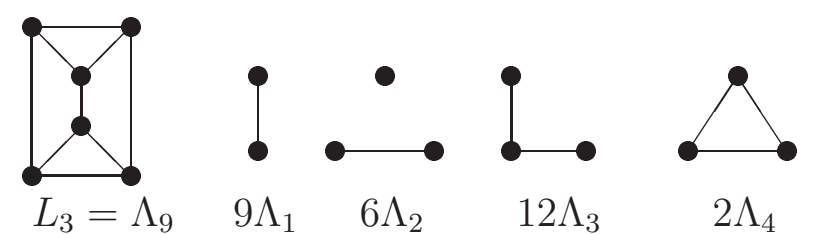

$9 \Lambda_{1}$

$6 \Lambda_{2}$

$12 \Lambda_{3}$
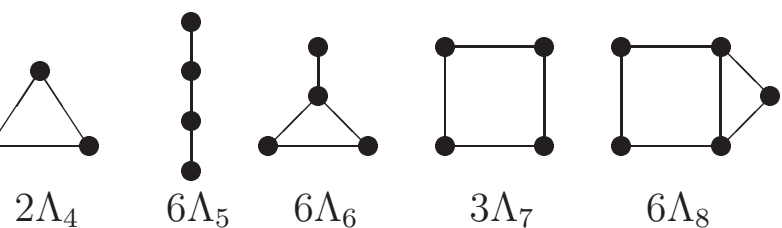

$3 \Lambda_{7}$

$6 \Lambda_{8}$

Figure 1: $L_{3}$ and its induced subgraphs.

The rows and the columns of $\mathcal{N}$-matrix of $L_{3}$ are both indexed by $\Lambda_{1}$ to $\Lambda_{9}$. The $\mathcal{N}$-matrix of $L_{3}$ is shown below.

$$
\mathcal{N}\left(L_{3}\right)=\left(\begin{array}{ccccccccc}
1 & 0 & 0 & 0 & 0 & 0 & 0 & 0 & 0 \\
1 & 1 & 0 & 0 & 0 & 0 & 0 & 0 & 0 \\
2 & 0 & 1 & 0 & 0 & 0 & 0 & 0 & 0 \\
3 & 0 & 0 & 1 & 0 & 0 & 0 & 0 & 0 \\
3 & 2 & 2 & 0 & 1 & 0 & 0 & 0 & 0 \\
4 & 1 & 2 & 1 & 0 & 1 & 0 & 0 & 0 \\
4 & 0 & 4 & 0 & 0 & 0 & 1 & 0 & 0 \\
6 & 3 & 6 & 1 & 2 & 2 & 1 & 1 & 0 \\
9 & 6 & 12 & 2 & 6 & 6 & 3 & 6 & 1
\end{array}\right)
$$


Let us associate an edge labelled poset with the graph $G$. Define a partial order $\preceq$ on the set $\Lambda(G)$ as follows: $\Lambda_{j} \preceq \Lambda_{k}$ if and only if $\Lambda_{j}$ is an induced subgraph of $\Lambda_{k}$. This poset is denoted by $(\Lambda(G), \preceq)$. We make the poset $(\Lambda(G), \preceq)$ an edge labelled poset by assigning a positive integer to every edge of its Hasse diagram, such that if $\Lambda_{k}$ covers $\Lambda_{j}$ then the edge label on $\Lambda_{j}-\Lambda_{k}$ is $\left(\begin{array}{c}\Lambda_{k} \\ \Lambda_{j}\end{array}\right)$. We say that two edge labelled posets are isomorphic if they are isomorphic as posets, and there is an isomorphism between them that preserves the edge labels. This naturally leads to the notion of the abstract edge labelled poset of $G$ : it is the isomorphism class of the edge labelled poset of $G$. Note that the notion of the abstract edge labelled poset of a graph is not to be confused with the isomorphism class of the Hasse diagram as a graph. An isomorphism from an edge labelled poset to itself is called an automorphism of the edge labelled poset. We denote the abstract edge labelled poset of $G$ by $\mathcal{E} \mathcal{L} \mathcal{P}(G)$. The Hasse diagram of the abstract edge labelled poset is simply the Hasse diagram of the edge labelled poset of $G$ with labels $\Lambda_{i}$ removed. The Hasse diagram of $\mathcal{E} \mathcal{L} \mathcal{P}\left(L_{3}\right)$ is shown in Figure 2.

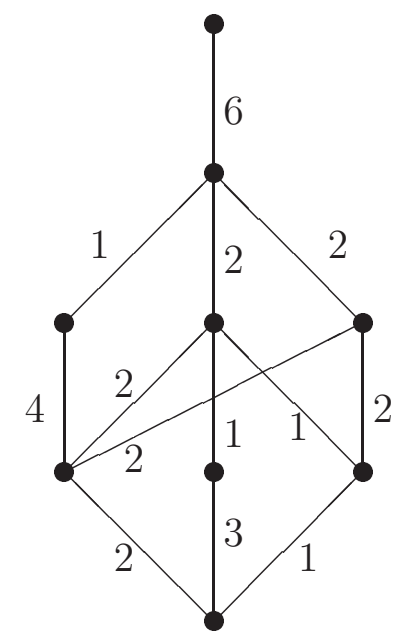

Figure 2: The abstract edge labelled poset of $L_{3}$.

Lemma 2.1. There is a rank function on $\rho$ on $\mathcal{E} \mathcal{L} \mathcal{P}(G)$ such that $\rho\left(\Lambda_{i}\right)=\rho\left(\Lambda_{j}\right)+1$ whenever $\Lambda_{i}$ covers $\Lambda_{j}$.

Proof. Each $\Lambda_{i}$ in $\Lambda(G)$ is nonempty. Therefore, for each $\Lambda_{i}$ in $\Lambda(G)$ and for each $k$ such that $2 \leq k \leq v\left(\Lambda_{i}\right)$ there is at least one nonempty induced subgraph $\Lambda_{j}$ of $\Lambda_{i}$ such that $v\left(\Lambda_{j}\right)=k$. Moreover, empty induced subgraphs do not belong to $\Lambda(G)$. Therefore, $\rho\left(\Lambda_{i}\right)=v\left(\Lambda_{i}\right)$ meets the requirements of a rank function.

Stanley [12] defines a rank function such that the $\rho(x)=0$ for a minimal element $x$. But we have deviated from that convention since $\rho\left(\Lambda_{i}\right)=v\left(\Lambda_{i}\right)$ for each $\Lambda_{i} \in \Lambda(G)$ is more 
convenient here. We now demonstrate that $\mathcal{N}(G)$ and $\mathcal{E} \mathcal{L} \mathcal{P}(G)$ are really equivalent, that is, they can be constructed from each other.

Lemma 2.2. Let $F$ and $H$ be two graphs, and let $q$ be an integer such that $v(F) \leq q \leq$ $v(H)$. Then

$$
\sum_{X \mid v(X)=q}\left(\begin{array}{c}
H \\
X
\end{array}\right)\left(\begin{array}{c}
X \\
F
\end{array}\right)=\left(\begin{array}{c}
v(H)-v(F) \\
q-v(F)
\end{array}\right)\left(\begin{array}{c}
H \\
F
\end{array}\right)
$$

where the summation is over distinct isomorphism types $X$.

Proof. This is similar to Kelly's Lemma 1.2. Each induced subgraph of $H$ that is isomorphic to $F$ is also an induced subgraph of $\left(\begin{array}{c}v(H)-v(F) \\ q-v(F)\end{array}\right)$ induced subgraphs of $H$ that have $q$ vertices.

Lemma 2.3. The structures $\mathcal{N}(G)$ and $\mathcal{E} \mathcal{L} \mathcal{P}(G)$ can be constructed from each other.

Proof. We first show how $\mathcal{N}(G)$ is constructed from $\mathcal{E} \mathcal{L} \mathcal{P}(G)$. The matrix $\mathcal{N}(G)$ is an $I \times I$ matrix where $I$ is the number of points in $\mathcal{E} \mathcal{L P}(G)$. Without the loss of generality, suppose that the points of $\mathcal{E} \mathcal{L} \mathcal{P}(G)$ are labelled from $\Lambda_{1}$ to $\Lambda_{I}$ such that if $\rho\left(\Lambda_{i}\right)<\rho\left(\Lambda_{j}\right)$ then $i<j$, where $\rho$ is the rank function defined in Lemma 2.1. Correspondingly, the rows and the columns of $\mathcal{N}(G)$ are indexed from $\Lambda_{1}$ to $\Lambda_{I}$. The edge labels in $\mathcal{E} \mathcal{L} \mathcal{P}(G)$ immediately give some of the entries in $\mathcal{N}(G)$ : if $\Lambda_{i}$ covers $\Lambda_{j}$ then $N_{i j}$ is the label on the edge joining $\Lambda_{i}$ and $\Lambda_{j}$. The diagonal entries are 1. Except $N_{11}$, all the other entries in the first row are 0 . We construct the remaining entries of $\mathcal{N}(G)$ by induction on the rank. The base case is rank 2. It corresponds to the first row, and is already filled. Let $f(r)$ denote the number of points of $\mathcal{E} \mathcal{L P}(G)$ that have rank at most $r$. Suppose now that the first $f(r)$ rows of $\mathcal{N}(G)$ are filled for some $r \geq 2$. Let $\Lambda_{i}$ be a graph of rank $r+1$, and let $\Lambda_{j}$ be a graph of rank at most $r$. Then $N_{i j}$ is computed by applying Lemma 2.2 with $q=r$.

$$
\sum_{\Lambda_{k} \mid \rho\left(\Lambda_{k}\right)=r}\left(\begin{array}{c}
\Lambda_{i} \\
\Lambda_{k}
\end{array}\right)\left(\begin{array}{c}
\Lambda_{k} \\
\Lambda_{j}
\end{array}\right)=\left(\begin{array}{c}
v\left(\Lambda_{i}\right)-v\left(\Lambda_{j}\right) \\
r-v\left(\Lambda_{j}\right)
\end{array}\right)\left(\begin{array}{c}
\Lambda_{i} \\
\Lambda_{j}
\end{array}\right)=\left(r+1-v\left(\Lambda_{j}\right)\right) N_{i j}
$$

On the LHS, $\left(\begin{array}{l}\Lambda_{k} \\ \Lambda_{j}\end{array}\right)$ are known by induction hypothesis. Since $\Lambda_{k}$ are the graphs covered by $\Lambda_{i},\left(\begin{array}{c}\Lambda_{i} \\ \Lambda_{k}\end{array}\right)$ are the edge labels. Therefore, $N_{i j}$ can be computed. This completes the construction of $\mathcal{N}(G)$ from $\mathcal{E} \mathcal{L} \mathcal{P}(G)$.

To construct $\mathcal{E} \mathcal{L} \mathcal{P}(G)$ from $\mathcal{N}(G)$, define a partial order $\preceq$ on $\left\{\Lambda_{1}, \Lambda_{2}, \ldots, \Lambda_{I}\right\}$ as follows: $\Lambda_{j} \preceq \Lambda_{i}$ if $N_{i j} \neq 0$. In this poset, if $\Lambda_{i}$ covers $\Lambda_{j}$ then assign an edge label $N_{i j}$ to the edge $\Lambda_{j}-\Lambda_{i}$ of the Hasse diagram of the poset. This completes the construction of $\mathcal{E} \mathcal{L} \mathcal{P}(G)$ from $\mathcal{N}(G)$.

Lemma 2.4. Given $\mathcal{N}(G), v\left(\Lambda_{i}\right)$ and $e\left(\Lambda_{i}\right)$ can be counted for each graph in $\Lambda(G)$. 
Proof. There is a unique row in $\mathcal{N}(G)$ that has only one nonzero entry (the diagonal entry 1). This row corresponds to $\Lambda_{1} \cong K_{2}$, and we assume it to be the first row. Now $e\left(\Lambda_{i}\right)=N_{i 1}$ for each $\Lambda_{i}$.

By Lemma 2.3, $\mathcal{E} \mathcal{L P}(G)$ is uniquely constructed. By Lemma 2.1, the rank function of the poset defined by $\rho\left(\Lambda_{1}\right)=2$ gives $v\left(\Lambda_{i}\right)=\rho\left(\Lambda_{i}\right)$ for each $\Lambda_{i}$.

Now on, without the loss of generality, we will assume that the nonisomorphic induced subgraphs $\Lambda_{1}, \Lambda_{2}, \ldots, \Lambda_{I}$ of a graph $G$ under consideration are ordered so that $v\left(\Lambda_{i}\right)$ are in a non-decreasing order. The first row will correspond to $\Lambda_{1} \cong K_{2}$ and the last row to $\Lambda_{I} \cong G$.

Lemma 2.5. The collection $\{\mathcal{N}(G-u) \mid u \in V G, e(G-u)>0\}$ is unambiguously determined by $\mathcal{N}(G)$.

Note that this collection is a "multiset", that is, an $\mathcal{N}$-matrix may appear multiple times in the collection.

Proof. Let $j \neq I$. The graph $\Lambda_{j}$ is a vertex deleted subgraph of $G$ if and only if for all $i \neq j \neq I, N_{i j}=0$. Now $N\left(\Lambda_{j}\right)$ is obtained by deleting $k$ 'th row and $k$ 'th column for each $k$ such that $N_{j k}=0$. A multiplicity $N_{I j}$ is assigned to $N\left(\Lambda_{j}\right)$. Equivalently, we can construct $\mathcal{E} \mathcal{L P}(G)$ by Lemma 2.3 , then construct the down set $\mathcal{E} \mathcal{L} \mathcal{P}\left(\Lambda_{j}\right)$ of each $\Lambda_{j}$ that is covered by $\Lambda_{I}=G$, and then construct $\mathcal{N}\left(\Lambda_{j}\right)$, and assign it a multiplicity equal to the edge label on $\Lambda_{I}-\Lambda_{j}$.

Remark It is is possible that for distinct $j$ and $k$, the matrices $N\left(\Lambda_{j}\right)$ and $N\left(\Lambda_{k}\right)$ are equal. In this case a multiplicity $N_{I j}$ is assigned to $N\left(\Lambda_{j}\right)$ and $N_{I k}$ is assigned to $N\left(\Lambda_{k}\right)$ while constructing the above collection.

Lemma 2.6. Let $r K_{1}$ be the $r$-vertex empty graph. The number of induced subgraphs of $G$ isomorphic to $r K_{1}$ is determined by $\mathcal{N}(G)$.

Proof. The required number is

$$
\left(\begin{array}{c}
G \\
r K_{1}
\end{array}\right)=\left(\begin{array}{c}
v(G) \\
r
\end{array}\right)-\sum_{j \mid v\left(\Lambda_{j}\right)=r} N_{I j}
$$

where indices $j$ in the summation are determined by Lemma 2.4 .

We are interested in the question of reconstructing a graph $G$ or some of its invariants given $\mathcal{N}(G)$. As indicated earlier, we will assume that the induced subgraphs $\Lambda_{i} ; i \in[1, I]$ are not given. We have the following relationship between Ulam's conjecture and the $\mathcal{N}$-matrix reconstructibility.

Proposition 2.7. Ulam's conjecture is true if and only if all graphs on three or more vertices are $\mathcal{N}$-matrix reconstructible. 
Proof. Proof of if: by Lemma 2.2, $\mathcal{N}(G)$ is constructed from $\mathcal{V} \mathcal{D}(G)$. Therefore, Ulam's conjecture is true if all graphs are $\mathcal{N}$-matrix reconstructible. In fact, a graph is reconstructible if it is $\mathcal{N}$-matrix reconstructible.

Proof of only if: this is proved by induction on the number of vertices. Let Ulam's conjecture be true. Since $N_{i 1}=e\left(\Lambda_{i}\right)$ for all $i$, every non-empty three vertex graph is $\mathcal{N}$-matrix reconstructible. Now, let all graphs on at most $n$ vertices, where $n \geq 3$, be $\mathcal{N}$ matrix reconstructible. Let $G$ be a graph on $n+1$ vertices. By Lemma 2.5, the collection $\{\mathcal{N}(G-u) ; u \in V G, e(G-u)>0\}$ is unambiguously determined by $\mathcal{N}(G)$. The number of empty graphs in $\mathcal{V} \mathcal{D}(G)$ is 0 or 1 , and is determined by Lemma 2.6. Therefore, by induction hypothesis, $\mathcal{V D}(G)$ is uniquely determined. Now the result follows from the assumption that Ulam's conjecture is true.

Since $\mathcal{N}(G)$ and $\mathcal{E} \mathcal{L} \mathcal{P}(G)$ are equivalent by Lemma 2.3, we rephrase Proposition 2.7 as follows.

Proposition 2.8. Ulam's conjecture is true if and only if all graphs on three or more vertices are reconstructible from their abstract edge labelled posets.

We would like to point out that reconstructing $G$ from $\mathcal{N}(G)$ or from $\mathcal{E} \mathcal{L P}(G)$ is not proved to be equivalent to reconstructing $G$ from $\mathcal{V D}(G)$. This poses a difficulty. For example, proving $\mathcal{N}$-matrix reconstructibility of disconnected graphs is as hard as Ulam's conjecture, although disconnected graphs are known to be vertex reconstructible. This is proved below.

For graphs $X$ and $Y$, we use the notation $X+Y$ to denote a graph that is a disjoint union of two graphs isomorphic to $X$ and $Y$, respectively. Suppose $G$ and $H$ are connected graphs having the same vertex deck. Consider graphs $2 G=G+G$ and $2 H=H+H$.

Lemma 2.9. Let $F$ be a graph on fewer than $2 v(G)$ vertices. If $F$ has a component isomorphic to $G$ (in which case we write $F=G+X)$ then $\left(\begin{array}{c}2 G \\ G+X\end{array}\right)=\left(\begin{array}{c}2 H \\ H+X\end{array}\right)$. If $F$ has no component isomorphic to $G$ then $\left(\begin{array}{c}2 G \\ F\end{array}\right)=\left(\begin{array}{c}2 H \\ F\end{array}\right)$.

Proof. When $F=G+X, X$ must have fewer than $v(G)-1$ vertices. Since $G$ and $H$ have identical vertex decks, by Kelly's Lemma 1.2, $\left(\begin{array}{c}G \\ X\end{array}\right)=\left(\begin{array}{c}H \\ X\end{array}\right)$. Therefore, $\left(\begin{array}{c}2 G \\ G+X\end{array}\right)=$ $\left(\begin{array}{c}2 H \\ H+X\end{array}\right)$

When $F$ does not have a component isomorphic to $G$ or $H$, then if $F$ has a component on $v(G)$ vertices then $\left(\begin{array}{c}2 G \\ F\end{array}\right)=\left(\begin{array}{c}2 H \\ F\end{array}\right)=0$. Therefore, assume that all components of $F$ have at most $v(G)-1$ vertices. Any realisation of $F$ as an induced subgraph of $2 G$ is a disjoint union of graphs isomorphic to $X$ and $Y$ such that $X$ is an induced subgraph of one component of $2 G$ and $Y$ is an induced subgraph of the other component of $2 G$. 
Moreover, $v(X)<v(G)$ and $v(Y)<v(G)$. Now $\left(\begin{array}{c}2 G \\ F\end{array}\right)=\left(\begin{array}{c}2 H \\ F\end{array}\right)$ follows from the fact that $G$ and $H$ have identical vertex decks and Kelly's Lemma.

The following corollary is an immediate consequence of the above lemma.

Corollary 2.10. Define a correspondence $f$ between $\Lambda(2 G)$ and $\Lambda(2 H)$ as follows.

1. $f(2 G)=2 H$

2. $F \in \Lambda(2 G)$ is not $2 G$ but has a component isomorphic to $G$. We write $F=G+X$, and set $f(F)=H+X$.

3. $F \in \Lambda(2 G)$ has no component isomorphic to $G$. In this case we set $f(F)=F$.

The correspondence defined above is a bijection.

Lemma 2.11. $\mathcal{N}(2 G)=\mathcal{N}(2 H)$.

Proof. For the bijection $f$ between non-empty induced subgraphs of $2 G$ and $2 H$ that was defined in Corollary 2.10, we show that, for any two nonisomorphic induced subgraphs $F_{1}$ and $F_{2}$ of $2 G$,

$$
\left(\begin{array}{l}
F_{2} \\
F_{1}
\end{array}\right)=\left(\begin{array}{l}
f\left(F_{2}\right) \\
f\left(F_{1}\right)
\end{array}\right)
$$

In view of Corollary 2.10, it is sufficient to show this when at least one of the graphs $F_{1}$ and $F_{2}$ has a component isomorphic to $G$.

1. When $F_{2}=2 G$, then Equation (10) follows from Lemma 2.9 and Corollary 2.10.

2. When $F_{1}=G+X$ and $F_{2}=G+Y$, and $v(X)<v(G)$ and $v(Y)<v(G)$, we have $\left(\begin{array}{l}F_{2} \\ F_{1}\end{array}\right)=\left(\begin{array}{l}Y \\ X\end{array}\right)=\left(\begin{array}{l}H+Y \\ H+X\end{array}\right)=\left(\begin{array}{l}f\left(F_{2}\right) \\ f\left(F_{1}\right)\end{array}\right)$.

3. $F_{2}=G+Z, v(Z)<v(G)$, but $F_{1}$ has no component isomorphic to $G$. In this case, any realisation of $F_{1}$ as an induced subgraph of $F_{2}$ may be represented (possibly in many ways) as $F_{1}=X+Y$ where $X$ is an induced proper subgraph of the component $G$ of $F_{2}$ and $Y$ is an induced subgraph of $Z$. Moreover, $v(X)<v(G)$ and $v(Y)<v(G)$. Since $\left(\begin{array}{c}G \\ X\end{array}\right)=\left(\begin{array}{c}H \\ X\end{array}\right)$, we have $\left(\begin{array}{c}G+Z \\ F_{1}\end{array}\right)=\left(\begin{array}{c}H+Z \\ f\left(F_{1}\right)\end{array}\right)$. Note that the actual value of $\left(\begin{array}{c}F_{2} \\ F_{1}\end{array}\right)$ may be written by considering all possible ways of realising $F_{1}$ as an induced subgraph of $G+Z$.

Thus we have shown Equation (10) for arbitrary non-empty induced subgraphs of $2 G$, which implies the result.

Theorem 2.12. Ulam's conjecture is true if and only if disconnected graphs on three or more vertices are $\mathcal{N}$-matrix reconstructible. 
Proof. The only if part follows from Proposition 2.7. The if part is proved by contradiction. Suppose $G$ and $H$ are connected nonisomorphic graphs with the same vertex deck, that is, they are a counter example to Ulam's conjecture. Then $2 G$ and $2 H$ are nonisomorphic but $\mathcal{N}(2 G)=\mathcal{N}(2 H)$ by Lemma 2.11. Therefore, $2 G$ and $2 H$ are disconnected graphs that are not $\mathcal{N}$-matrix reconstructible.

The following result is proved along the lines of Lemma 2.11.

Theorem 2.13. Ulam's conjecture is true if and only if the edge labelled poset of each graph has only the trivial automorphism.

Proof. The proof of only if is done by contradiction. Suppose that $\mathcal{E} \mathcal{L P}(G)$ has a nontrivial automorphism $\sigma$. Then there are nonisomorphic induced subgraphs $\Lambda_{i}$ and $\Lambda_{j}$ of $G$ such that $\sigma\left(\Lambda_{i}\right)=\Lambda_{j}$. The downsets (or the edge labelled posets) of $\Lambda_{i}$ and $\Lambda_{j}$ must be isomorphic. Therefore, by Proposition 2.8, there is a counter example to Ulam's conjecture.

The proof of if is also done by contradiction. Suppose that Ulam's conjecture is false, and $G$ and $H$ are connected nonisomorphic graphs having identical vertex decks. We show that $\mathcal{E} \mathcal{L} \mathcal{P}(G+H)$ has a nontrivial automorphism. Define a bijective map $\sigma: \Lambda(G+H) \rightarrow \Lambda(G+H)$ as follows.

1. The graph $G+H$ is mapped to itself.

2. If $\Lambda_{i} \in \Lambda(G+H)$ has a component isomorphic to $G$, then denote $\Lambda_{i}$ by $G+X$, where $X$ is a proper subgraph of the component isomorphic to $H$. In this case, set $\sigma(G+X)=H+X$.

3. If $\Lambda_{i}$ is $H+X$, where $X$ is a proper subgraph of the component isomorphic to $G$, then set $\sigma(H+X)=G+X$.

4. For all other graphs $\Lambda_{i} \in \Lambda(G+H), \sigma\left(\Lambda_{i}\right)=\Lambda_{i}$.

We now show that $\sigma$ is an automorphism of $\mathcal{E} \mathcal{L P}(G+H)$. That is, we show that $\left(\begin{array}{c}\Lambda_{i} \\ \Lambda_{j}\end{array}\right)=\left(\begin{array}{c}\sigma\left(\Lambda_{i}\right) \\ \sigma\left(\Lambda_{j}\right)\end{array}\right)$ for any two graphs $\Lambda_{i}$ and $\Lambda_{j}$ in $\Lambda(G+H)$.

We have to consider only the case in which at least one of $\Lambda_{i}$ and $\Lambda_{j}$ has a component isomorphic to $G$ or $H$, and $v\left(\Lambda_{j}\right) \leq v\left(\Lambda_{i}\right)$.

1. $\Lambda_{j}=G+X$ and $\Lambda_{i}=G+H$. In this case,

$$
\left(\begin{array}{l}
G+H \\
G+X
\end{array}\right)=\left(\begin{array}{l}
H \\
X
\end{array}\right)=\left(\begin{array}{l}
G \\
X
\end{array}\right)=\left(\begin{array}{l}
H+G \\
H+X
\end{array}\right)=\left(\begin{array}{c}
\sigma(G+H) \\
\sigma(G+X)
\end{array}\right) .
$$

2. $\Lambda_{j}=G+X$ and $\Lambda_{i}=G+Y$ and $v(Y)<v(G)=v(H)$. In this case,

$$
\left(\begin{array}{l}
G+Y \\
G+X
\end{array}\right)=\left(\begin{array}{l}
Y \\
X
\end{array}\right)=\left(\begin{array}{l}
H+Y \\
H+X
\end{array}\right)=\left(\begin{array}{c}
\sigma(G+Y) \\
\sigma(G+X)
\end{array}\right) \text {. }
$$


3. $\Lambda_{j}=G+X$ and $\Lambda_{j}=H+Y$ and $Y \nsucceq G$. In this case,

$$
\left(\begin{array}{l}
H+Y \\
G+X
\end{array}\right)=\left(\begin{array}{c}
G+Y \\
H+X
\end{array}\right)=0
$$

4. $\Lambda_{j}=G+X$ and $\Lambda_{i}$ has no component isomorphic to $G$ or $H$. In this case, $\left(\begin{array}{c}\Lambda_{i} \\ G+X\end{array}\right)=\left(\begin{array}{c}\Lambda_{i} \\ H+X\end{array}\right)=0$

5. $\Lambda_{j}$ has no component isomorphic to $G$ or $H$, and $\Lambda_{i}=G+H$. This is trivial since $\sigma\left(\Lambda_{j}\right)=\Lambda_{j}$ and $\sigma(G+H)=G+H$

6. $\Lambda_{j}$ has no component isomorphic to $G$ or $H$ and $\Lambda_{i}=G+X$, where $v(X)<$ $v(G)=v(H)$. In this case, a realisation of $\Lambda_{j}$ as an induced subgraph of $G+X$ may be written as $\Lambda_{j}=Y+Z$, where $Y$ is an induced subgraph of $G$ and $Z$ is an induced subgraph of $X$. Since, $\left(\begin{array}{l}G \\ Y\end{array}\right)=\left(\begin{array}{l}H \\ Y\end{array}\right)$, the number of such realisations is $\left(\begin{array}{c}G \\ Y\end{array}\right)\left(\begin{array}{l}X \\ Z\end{array}\right)=\left(\begin{array}{l}H \\ Y\end{array}\right)\left(\begin{array}{l}X \\ Z\end{array}\right)$. By summing over all possible ways of realising $\Lambda_{j}$ as an induced subgraph of $G+X$, we get $\left(\begin{array}{c}G+X \\ \Lambda_{j}\end{array}\right)=\left(\begin{array}{c}H+X \\ \Lambda_{j}\end{array}\right)$.

7. All the above arguments are valid when $G$ and $H$ are interchanged.

Thus we have constructed a non-trivial automorphism, completing the if part.

We conclude this section with a result on trees.

Theorem 2.14. Trees and forests are $\mathcal{N}$-matrix reconstructible.

Proof. The class of simple acyclic graphs is closed under vertex deletion. Therefore, we can use the method in the proof of Proposition 2.7. Let $T$ be a tree or a non-empty forest on three or more vertices. We prove by induction on $v(T)$ that $T$ is uniquely reconstructible from $\mathcal{N}(T)$. The base case is $v(T)=3$. All graphs on 3 vertices are $\mathcal{N}$-matrix reconstructible by Lemma 2.4. Suppose each acyclic graphs on at most $k$ can be recognised and reconstructed from its $\mathcal{N}$-matrix. Let $v(T)=k+1$. By Lemma 2.5, the collection $\{\mathcal{N}(T-u) \mid u \in V T\}$ is unambiguously determined. Then by induction hypothesis, $T-u$ are determined (along with their multiplicities). The subgraphs in the vertex deck that are not determined by Lemma 2.5 are the ones having no edges. Since Ulam's conjecture has been proved for trees and disconnected simple graphs in [7], $T$ is $\mathcal{N}$-matrix reconstructible.

Remark If Ulam's conjecture is true for a class of graphs that is closed under vertex deletion, then the class is also $\mathcal{N}$-matrix reconstructible. 


\section{Tutte-Kocay theory on the $\mathcal{N}$-matrix.}

In this section we will compute several invariants of a graph $G$ from its $\mathcal{N}$-matrix. The invariants include the number of spanning trees, the number of spanning unicyclic subgraphs containing a cycle of specified length, the characteristic polynomial and the rank polynomial.

An outline of the proof. First we outline how the above mentioned invariants are calculated from the vertex deck using Kocay's Lemma.

1. Suppose the graphs $F_{1}, F_{2}, \ldots, F_{k}$ satisfy $\sum_{i} v\left(F_{i}\right)=v(G)$ and $v\left(F_{i}\right)<v(G) \forall i$. Kocay's Lemma then gives the number of disconnected spanning subgraphs having components isomorphic to $F_{1}, F_{2}, \ldots, F_{k}$.

2. Kacay's lemma is then applied to $F_{1}=F_{2}=\ldots=F_{k}=K_{2}$, where $k=v(G)-1$. Since disconnected spanning subgraphs of each type are counted in the first step, we can now count the number of spanning trees.

3. The second step is repeated with $k=v(G)$. Since the number of spanning trees and disconnected spanning subgraphs of each type are known from the first two steps, we can now count the number of hamiltonian cycles.

4. Once the above three steps are completed, many other invariants, such as the characteristic polynomial, rank polynomial, etc. are easily computed.

The procedure outlined above cannot be implemented on the $\mathcal{N}$-matrix in a straight forward manner. We do not know all the induced proper subgraphs. But we observe that the above procedure essentially reduces counting certain spanning subgraphs to counting them on vertex proper subgraphs. It turns out that we do not really need the number of vertex proper subgraphs of each type. We only need to know the 'cycle structure', that is, $\psi_{i}\left(\Lambda_{j}\right)$ for each $i \leq v\left(\Lambda_{j}\right)$, for each $j<I$. Next we outline the strategy to construct the cycle structure.

Suppose $X, Y, \ldots$ is a list of some graph invariants that are either polynomials or numbers, for example, the number of hamiltonian cycles in a graph or the chromatic polynomial of a graph. We say that an invariant $Z$ can be reduced to invariants $X, Y, \ldots$ (or $Z$ has a reduction on the $\mathcal{N}$-matrix) if for each graph $G$ having a non-empty edge set,

1. $Z(G)$ can be written as $Z(G)=\Theta\left(X\left(G_{U}\right), Y\left(G_{V}\right), \ldots\right)$ where $\Theta(x, y, \ldots)$ is a polynomial in $x, y, \ldots$, and $U, V, \ldots$ are proper subsets of $V G$.

2. the coefficient of each term in the polynomial can be computed from $\mathcal{N}(G)$.

Proving an identity that gives a reduction of an invariant $Z$ as in the above equation is not in itself sufficient to claim that $Z$ is $\mathcal{N}$-matrix reconstructible. If the invariants $X_{1}, X_{2}, \ldots, X_{k}$ appear on the RHS of the above equation, then it is essential to show that the invariants $X_{1}, X_{2}, \ldots, X_{k}$ themselves can be reduced to $X_{1}, X_{2}, \ldots, X_{k}$. The reconstructibility of $Z$ and $X_{1}, X_{2}, \ldots, X_{k}$ from the $\mathcal{N}$-matrix is then proved by induction 
on $v(G)$. That is possible because of the requirement that the sets $U, V, \ldots$ are proper subsets of $V G$. It is worth noting that the chromatic polynomial computation given in Biggs [2] essentially follows a similar style. In several lemmas that precede the main theorem, we will prove identities of the form $Z(G)=\Theta\left(X\left(G_{U}\right), Y\left(G_{V}\right), \ldots\right)$. It will become clear that in the end all invariants computed here will reduce to the cycle structure of proper subgraphs.

Lemma 3.1. For $i<v(G)$, the number of cycles of length $i$ in $G$ has a reduction on $\mathcal{N}(G)$ given by

$$
\psi_{i}(G)=\frac{1}{v(G)-i} \sum_{u \in V G} \psi_{i}(G-u)=\frac{1}{v(G)-i} \sum_{j \mid v\left(\Lambda_{j}\right)=v(G)-1} N_{I j} \psi_{i}\left(\Lambda_{j}\right)
$$

Proof. This immediately follows from Kelly's Lemma 1.2 and Lemma 2.5.

Definition 3.2. Let $X$ be a subset of $V G$. Let $A \equiv\left(a_{i}\right)_{i=1}^{k}$ be a sequence in $[2,|X|]$. A $k$-tuple of cycles in $G_{X}$, corresponding to the sequence $A$, is a $k$-tuple of cycles in $G_{X}$ such that the $k$ cycles have lengths $a_{1}, a_{2}, \ldots, a_{k}$, respectively. Additionally, if the cycles in the $k$-tuple span the set $X$, then it is called a spanning cycle cover of $G_{X}$, corresponding to the sequence $A$. The number of $k$-tuples of cycles in $G_{X}$, corresponding to the sequence $A$, is denoted by $p\left(A \rightarrow G_{X}\right)$. The number of spanning cycle covers of $G_{X}$, corresponding to the sequence $A$, is denoted by $c\left(A \rightarrow G_{X}\right)$.

\section{Lemma 3.3.}

$$
p\left(A \rightarrow G_{X}\right)=\sum_{Y \subseteq X} c\left(A \rightarrow G_{Y}\right)
$$

Proof.

$$
\begin{aligned}
p\left(A \rightarrow G_{X}\right) & =\prod_{j=1}^{k} \psi_{a_{j}}\left(G_{X}\right) \\
& =\left|\left\{\left(F_{1}, F_{2}, \ldots, F_{k}\right) \mid(\forall j \in[1, k])\left(F_{j} \subseteq G_{X}, F_{j} \cong C_{a_{j}}\right)\right\}\right| \\
& =\sum_{Y \subseteq X} c\left(A \rightarrow G_{Y}\right)
\end{aligned}
$$

Thus we have grouped together the $k$-tuples of cycles in groups that span each subset of $X$. This is essentially the idea of Kocay's Lemma 1.3.

Lemma 3.4. If $A \equiv\left(a_{i}\right)_{i=1}^{k}$ is a sequence in $[2, v(G)-1]$ then $p(A \rightarrow G)$ has a reduction on $\mathcal{N}(G)$ given by

$$
p(A \rightarrow G)=\prod_{i=1}^{k} \psi_{a_{i}}(G)=\prod_{i=1}^{k}\left(\frac{1}{v(G)-a_{i}} \sum_{j \mid v\left(\Lambda_{j}\right)=v(G)-1} N_{I j} \psi_{a_{i}}\left(\Lambda_{j}\right)\right)
$$

Proof. This follows from the definition of $p(A \rightarrow G)$ and Lemma 3.1. 
Lemma 3.5. If $A \equiv\left(a_{i}\right)_{i=1}^{k}$ is a sequence in $[2, v(G)-1]$ then $c(A \rightarrow G)$ has a reduction on $\mathcal{N}(G)$.

Proof. By Möbius inversion of Equation (12), we write

$$
c\left(A \rightarrow G_{X}\right)=\sum_{Y \subseteq X}(-1)^{|X \backslash Y|} p\left(A \rightarrow G_{Y}\right)
$$

which implies

$$
c(A \rightarrow G)=\sum_{j=1}^{I}(-1)^{v(G)-v\left(\Lambda_{j}\right)} N_{I j} p\left(A \rightarrow \Lambda_{j}\right)
$$

By Lemma 3.4, the RHS of this equation has a reduction on $\mathcal{N}(G)$. Therefore, $c(A \rightarrow G)$ has a reduction on $\mathcal{N}(G)$.

The following definition restricts the spanning cycle covers of Definition 3.2 to connected spanning cycle covers.

Definition 3.6. Let $X$ be a subset of $V G$. Let $A \equiv\left(a_{i}\right)_{i=1}^{k}$ be a sequence in $[2,|X|]$. A connected spanning cycle cover of $G_{X}$, corresponding to the sequence $A$, is a $k$ tuple of cycles in $G_{X}$ such that the $k$ cycles have lengths $a_{1}, a_{2}, \ldots, a_{k}$, respectively, and together they constitute a connected subgraph spanning the set $X$. More formally, it is a $k$-tuple $\left(F_{1}, F_{2}, \ldots, F_{k}\right)$ such that $(\forall j \in[1, k])\left(F_{j} \subseteq G_{X}, F_{j} \cong C_{a_{j}}\right), \cup_{j=1}^{k} V F_{j}=X$, and $\cup_{j=1}^{k} F_{j}$ is connected. The number of connected spanning cycle covers of $G_{X}$, corresponding to the sequence $A$, is denoted by $\operatorname{con}\left(A \rightarrow G_{X}\right)$. The disconnected spanning cycle covers are defined similarly, and their number, corresponding to a sequence $A$, is denoted by $\operatorname{discon}\left(A \rightarrow G_{X}\right)$.

Let $A \equiv\left(A_{i}\right)_{i=1}^{l}$ be a list of $l$ non-increasing sequences such that $A_{i} \equiv\left(a_{i j}\right)_{j=1}^{k_{i}}$; $a_{i j} \in[2, v(G)]$. Let $B \equiv\left(b_{i}\right)_{i=1}^{l}$ be a sequence in $[2, v(G)]$. Let $m \leq n$. We now define quantities $Q_{m}(A, B \rightarrow G)$ and $T_{p}(A, B \rightarrow G)$ that are based on connected spanning cycle covers as follows.

$$
\begin{aligned}
Q_{m}(A, B \rightarrow G) & =\sum_{\substack{S \subseteq V(G) \\
|S|=m}} \prod_{i=1}^{l}\left(\sum_{\substack{X \subseteq S \\
|X|=b_{i}}} \operatorname{con}\left(A_{i} \rightarrow G_{X}\right)\right) \\
& \left.=\sum_{\substack{S \subseteq V(G) \\
|S|=m}} \sum_{\substack{\left(X_{1}, X_{2}, \ldots, X_{l}\right)\left| \\
\bigcup_{j=1}^{l} X_{j} \subseteq S\\
\right| X_{j} \mid=b_{j} \forall j}}\left(\prod_{i=1}^{l} \operatorname{con}\left(A_{i} \rightarrow G_{X_{i}}\right)\right)\right) \\
& =\sum_{p \leq m} T_{p}(A, B \rightarrow G)\left(\begin{array}{c}
v(G)-p \\
m-p
\end{array}\right)
\end{aligned}
$$


where

$$
T_{p}(A, B \rightarrow G)=\sum_{\substack{\left(X_{1}, X_{2}, \ldots, X_{l}\right)\left| \\\cup_{j=1}^{l} X_{j} \subseteq V(G)\\\right| \cup_{j=1}^{l} X_{j}|=p\\| X_{j} \mid=b_{j} \forall j}}\left(\prod_{i=1}^{l} \operatorname{con}\left(A_{i} \rightarrow G_{X_{i}}\right)\right)
$$

Solving the system of equations for $T_{m}(A, B \rightarrow G)$, we can write

$$
T_{m}(A, B \rightarrow G)=\sum_{p \leq m}(-1)^{m-p}\left(\begin{array}{c}
v(G)-p \\
m-p
\end{array}\right) Q_{p}(A, B \rightarrow G)
$$

When $m=v(G)$, this is simply

$$
T_{v(G)}(A, B \rightarrow G)=\sum_{p \leq v(G)}(-1)^{v(G)-p} Q_{p}(A, B \rightarrow G)
$$

Note that if $m<\max _{i, j}\left(a_{i j}\right)$ for some $i, j$ then $T_{m}(A, B \rightarrow G)$ and $Q_{m}(A, B \rightarrow G)$ are both 0 .

Lemma 3.7. If $A_{i} ; i \in[1, l]$ are sequences in $[2, v(G)-1]$, and $B \equiv\left(b_{i}\right)_{i=1}^{l}$ are sequences in $[2, v(G)-1]$ then $Q_{m}(A, B \rightarrow G)$ and $T_{m}(A, B \rightarrow G)$ have reductions on the $\mathcal{N}$-matrix for each $m \leq v(G)$.

Proof. We write $Q_{m}(A, B \rightarrow G)$ in terms of $\Lambda_{j}$ and the entries of $\mathcal{N}(G)$.

$$
\begin{aligned}
Q_{m}(A, B \rightarrow G) & =\sum_{\substack{S \subseteq V(G) \\
|S|=m}} \prod_{i=1}^{l}\left(\sum_{\substack{X \subseteq S \\
|X|=b_{i}}} \operatorname{con}\left(A_{i} \rightarrow G_{X}\right)\right) \\
& =\sum_{p \mid v\left(\Lambda_{p}\right)=m} N_{I p} \prod_{i=1}^{l}\left(\sum_{j \mid v\left(\Lambda_{j}\right)=b_{i}} N_{p j} \operatorname{con}\left(A_{i} \rightarrow \Lambda_{j}\right)\right)
\end{aligned}
$$

Since $b_{i}<v(G)$, Equations $(21)$ reduce $Q_{m}(A, B \rightarrow G)$ to the invariants $\operatorname{con}\left(A_{i} \rightarrow \Lambda_{j}\right)$ for $m \leq v(G)$. Therefore, by Equation (19), $T_{m}(A, B \rightarrow G)$ are also reduced to the invariants $\operatorname{con}\left(A_{i} \rightarrow \Lambda_{j}\right)$ for each $m \leq v(G)$. Note that if $a_{i k}>v\left(\Lambda_{j}\right)$ for some $k$ then $\operatorname{con}\left(A_{i} \rightarrow \Lambda_{j}\right)=0$.

Lemma 3.8. If $A \equiv\left(a_{i}\right)_{i=1}^{k}$ is a sequence in $[2, v(G)-1]$ then $\operatorname{con}(A \rightarrow G)$ has a reduction on $\mathcal{N}(G)$.

Proof. The idea of the proof is similar to Kocay's Lemma. First $c(A \rightarrow G)$ is written as $\operatorname{con}(A \rightarrow G)+\operatorname{discon}(A \rightarrow G)$. Then $\operatorname{discon}(A \rightarrow G)$ is expressed in terms of $\operatorname{con}\left(A_{i} \rightarrow G_{X_{i}}\right)$ where $X_{i}$ are proper subsets of $V G$, and $A_{i}$ are certain subsequences of 
$A$. Then $\operatorname{discon}(A \rightarrow G)$ are related to $T_{v(G)}\left(A^{P}, B \rightarrow G\right)$ for certain subsequences $A^{P}$ of $A$ and certain sequences $B$ constructed from appropriate partitions of $V G$. Since the reductions of $c(A \rightarrow G)$ and $T_{v(G)}\left(A^{P}, B \rightarrow G\right)$ have already been obtained, we get a reduction of $\operatorname{con}(A \rightarrow G)$.

Let $\mathcal{P}_{q}\left(N_{k}\right)$ be the set of all partitions of $N_{k}$ in $q$ parts. A partition $P$ in $\mathcal{P}_{q}\left(N_{k}\right)$, is denoted by $P=\left\{N_{k}^{1}, N_{k}^{2}, \ldots, N_{k}^{q}\right\}$. Consider an arbitrary $k$ tuple $\left(F_{1}, F_{2}, \ldots, F_{k}\right)$ such that $(\forall i \in[1, k])\left(F_{i} \subseteq G, F_{i} \cong C_{a_{i}}\right)$. It defines a partition $P \in \mathcal{P}_{q}\left(N_{k}\right)$ so that $h$ and $j$ are in the same part of $P$ if and only if $F_{h}$ and $F_{j}$ are subgraphs of the same connected component of $\cup_{i=1}^{k} F_{i}$. We denote the contribution to $c(A \rightarrow G)$ from such tuples by $c_{P}(A \rightarrow G)$, and write

$$
c(A \rightarrow G)=\sum_{q} \sum_{P \in \mathcal{P}_{q}} c_{P}(A \rightarrow G)
$$

Let the set of solutions to the equation $\sum_{i=1}^{q} b_{i}=v(G)$ be $\mathcal{B}(q)$. We can then write

$$
c(A \rightarrow G)=\sum_{q} \sum_{P \in \mathcal{P}_{q}} \sum_{B \in \mathcal{B}(q)} c_{P B}(A \rightarrow G)
$$

where one more suffix $B$ in the summand is used to denote those tuples for which the connected component corresponding to part $N_{k}^{i}$ has $b_{i}$ vertices, for $i \in[1, q]$. Now expanding the summand in terms of con(...), we get

$$
c(A \rightarrow G)=\sum_{q} \sum_{P \in \mathcal{P}_{q}} \sum_{B \in \mathcal{B}(q)} \sum_{\substack{\left.X_{1}, X_{2}, \ldots, X_{q}\right)\left| \\\cup_{j=1}^{q} X_{j}=V(G)\\\right| X_{j} \mid=b_{j} \forall j}} \prod_{i=1}^{q} \operatorname{con}\left(A_{i} \rightarrow G_{X_{i}}\right)
$$

where $A_{i}$ is the subsequence of $A$ with indexing set $N_{k}^{i}$. Innermost summation and product are now replaced by $T_{v(G)}\left(A^{P}, B \rightarrow G\right)$, so

$$
c(A \rightarrow G)=\sum_{q} \sum_{P \in \mathcal{P}_{q}} \sum_{B \in \mathcal{B}(q)} T_{v(G)}\left(A^{P}, B \rightarrow G\right)
$$

where $A^{P}$ is the collection of subsequences $A_{i}$ of $A ; i \in[1, q]$, corresponding to the partition $P$.

By Lemma 3.5, the LHS of Equation (25) has a reduction on $\mathcal{N}(G)$. By Lemma 3.7, each term on the RHS, except the term $\operatorname{con}(A \rightarrow G)$, which corresponds to $q=1$, has a reduction on $\mathcal{N}(G)$. This proves that $\operatorname{con}(A \rightarrow G)$ has a reduction on $\mathcal{N}(G)$.

Corollary 3.9. Let $\operatorname{tr}(G)$ be the number of spanning trees in $G$, and let uni $(G, r)$ be the number of spanning unicyclic subgraphs of $G$ containing an $r$-cycle, respectively. Then, $\operatorname{tr}(G)$ and uni $(G, r) ; r \in[3, v(G)]$ have reductions on $\mathcal{N}(G)$.

Proof. Define $A \equiv\left(a_{i}\right)_{i=1}^{v(G)-1}$ such that $a_{i}=2 \forall i \in[1, v(G)-1]$. By Lemma 3.8, con $(A \rightarrow$ $G)$ has a reduction on $\mathcal{N}(G)$. But $\operatorname{con}(A \rightarrow G)=(v(G)-1) ! \operatorname{tr}(G)$. Therefore, $\operatorname{tr}(G)$ has a reduction $\mathcal{N}(G)$. 
To reduce uni $(G, r) ; r \in[3, v(G)-1]$, define $A \equiv\left(a_{j}\right)_{j=1}^{v(G)-r+1}$ such that $a_{1}=r$, and $a_{j}=2 \forall j \in[2, v(G)-r+1]$. Again, $\operatorname{con}(A \rightarrow G)=(v(G)-r)$ !uni $(G, r)$ has a reduction $\mathcal{N}(G)$ by Lemma 3.8. Thus uni $(G, r)$ also has a reduction on $\mathcal{N}(G)$.

To reduce the number of hamiltonian cycles, let $A \equiv\left(a_{i}\right)_{i=1}^{v(G)} ; a_{i}=2 \forall i \in[1, v(G)]$. We have, $\operatorname{con}(A \rightarrow G)=(v(G)-1) ! S(v(G), v(G)-1) \operatorname{tr}(G)+\sum_{i=3}^{v(G)-1} v(G)$ ! uni $(G, i)+$ $v(G)$ !ham $(G)$, where $S(v(G), v(G)-1)$ is the Sterling number of the second kind computed for $(v(G), v(G)-1)$. Therefore, $\operatorname{ham}(G)$, has a reduction $\mathcal{N}(G)$.

Lemma 3.10. The coefficients $c_{i}(G)$ of the characteristic polynomial of $G$ are given by $c_{0}(G)=1$ and

$$
c_{i}(G)=\frac{1}{v(G)-i} \sum_{j \mid v\left(\Lambda_{j}\right)=v(G)-1} N_{I j} c_{i}\left(\Lambda_{j}\right) \text { for } 0<i<v(G) .
$$

Proof. By Lemma 1.7 we write $P^{\prime}(G ; \lambda)=\sum_{u \in V G} P(G-u ; \lambda)$. Equating identical powers of $\lambda$ on the two sides, we get the result.

Corollary 3.11. If $F$ is an elementary graph on $v(G)$ vertices then $\left[\begin{array}{c}G \\ F\end{array}\right]$ has a reduction on $\mathcal{N}(G)$.

Proof. The case when $F$ is a hamiltonian cycle is handled in Corollary 3.9. If $F$ is not a cycle, define a sequence $A \equiv\left(a_{i}\right)_{i=1}^{k}, a_{i} \in[2, v(G)-1]$ such that $\sum_{i=1}^{k} a_{i}=v(G)$. It is uniquely associated with an elementary graph $F \in L_{v(G)}$, so that the components of $F$ are cycles of length $a_{i}$, or $K_{2}$ if $a_{i}=2$. Now $c(A \rightarrow G)=c(A \rightarrow F)\left[\begin{array}{c}G \\ F\end{array}\right]$, and $c(A \rightarrow F)$ depends only on the multiplicity of each cycle length in $F$. By Lemma 3.5, $c(A \rightarrow G)$ has a reduction on the $\mathcal{N}$-matrix. Therefore, $\left[\begin{array}{c}G \\ F\end{array}\right]$ has a reduction on the $\mathcal{N}$-matrix.

The following chart shows how various invariants were reduced to other invariants. For example, it shows that $\operatorname{con}(A \rightarrow G) ; 2 \leq a_{i} \leq v(G)$ can be reduced to computing $\operatorname{ham}(G), \operatorname{con}(A \rightarrow G) ; 2 \leq a_{i}<v(G)$ and $p(A \rightarrow G) ; 2 \leq a_{i}<v(G)$. It is clear from the diagram that computing $\operatorname{con}(A \rightarrow G) ; 2 \leq a_{i} \leq v(G)$ and ham $(G)$ reduces to computing the same invariants for induced proper subgraphs. 


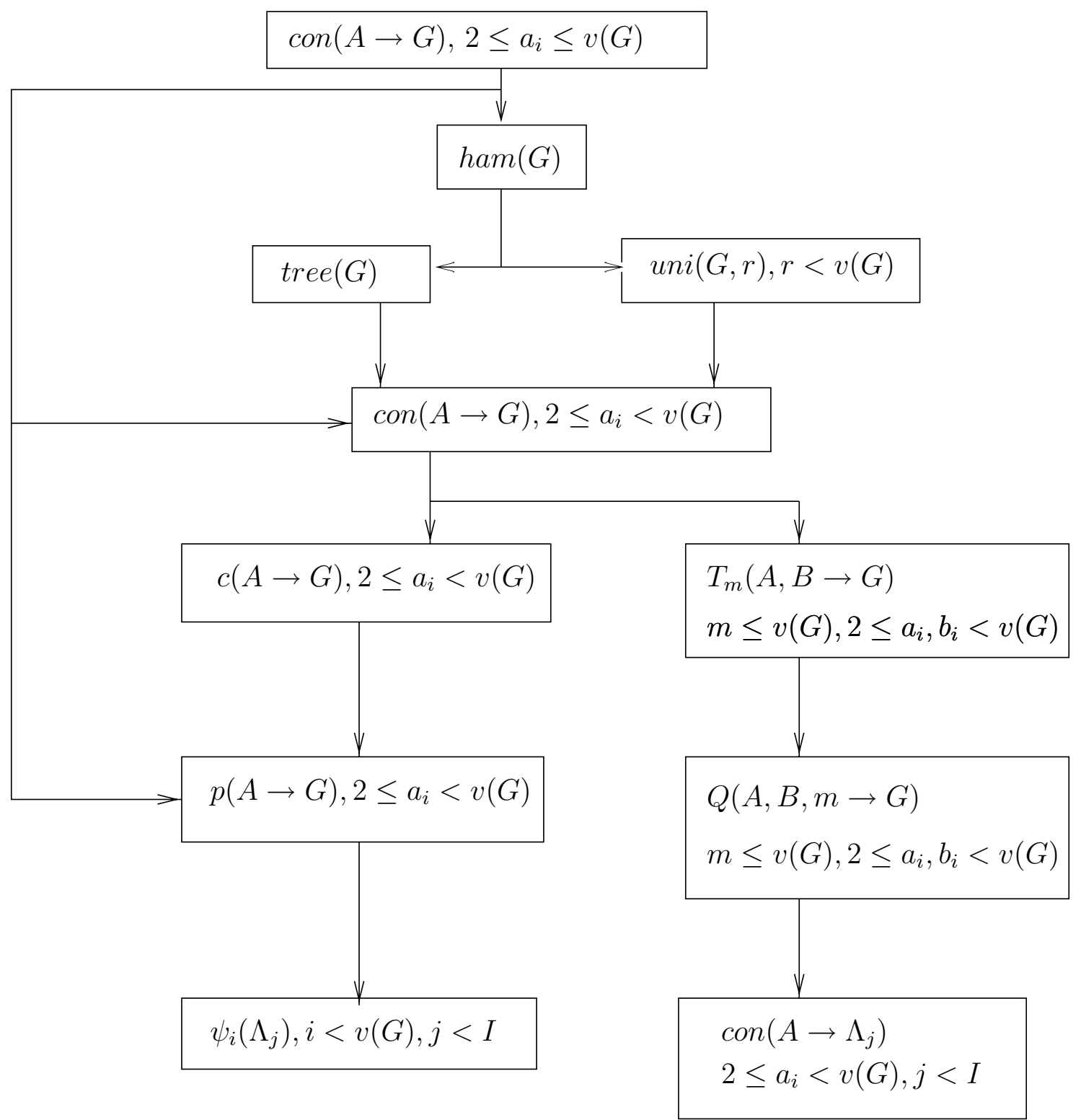

Figure 3: A summary of invariant reductions

This makes the reconstructibility of several invariants obvious.

Theorem 3.12. Suppose that $G$ is a simple finite graph, and we are given $\mathcal{N}(G)$.

1. Let $A \equiv\left(a_{i}\right)_{i=1}^{k}$ be a sequence such that $a_{i} \in[2, v(G)]$. Then $\operatorname{con}(A \rightarrow G)$ and $\operatorname{ham}(G)$ are reconstructible from $\mathcal{N}(G)$.

2. If $A_{i} ; i \in[1, l]$ are sequences in $[2, v(G)]$, and $B \equiv\left(b_{i}\right)_{i=1}^{l}$ is a sequence in $[2, v(G)]$ then $Q_{m}(A, B \rightarrow G)$ and $T_{m}(A, B \rightarrow G)$ are reconstructible from $\mathcal{N}(G)$ for each $m \leq v(G)$. 
3. the number of spanning trees in $G$, the number of cycles of length $i$, for $3 \leq i \leq$ $v(G)$, the number of unicyclic subgraphs containing a cycle of length $i$, for each $i \in[3, v(G)]$, and the characteristic polynomial $P(G ; \lambda)$ are all reconstructible from $\mathcal{N}(G)$.

Proof. We prove the first item by induction on $v(G)$. The base case is $v(G)=2$. In this case $G=K_{2}$. Now suppose that $\operatorname{con}(A \rightarrow G)$ and $\operatorname{ham}(G)$ are reconstructible from $\mathcal{N}(G)$ when $v(G)<s$ for an arbitrary sequence $A \equiv\left(a_{i}\right)_{i=1}^{k}$ of integers in $[2, v(G)]$. Now let $v(G)=s$. Lemmas and Corollaries 3.1 to 3.9 imply that computations of $\operatorname{con}(A \rightarrow G)$ and $\operatorname{ham}(G)$ reduce to computations on induced proper subgraphs of $G$, thus completing the induction step, and the proof of the first item.

Since all other intermediate invariants $T_{m}(\ldots), Q_{m}(\ldots)$, the number of spanning trees, the number of unicyclic graphs having a cycle of a specified length, number of cycles of each length, number of elementary spanning graphs of each type, etc. have been reduced to computations of invariants $\operatorname{con}\left(A \rightarrow \Lambda_{j}\right) ; j<I$ and $\psi_{i}\left(\Lambda_{j}\right) ; i<v(G), j<I$, the remaining parts of the theorem follow.

There is another way of proving the $\mathcal{N}$-matrix reconstructibility of the characteristic polynomial. Let $\bar{G}$ denote the complement of $G$. From $\mathcal{N}(G)$, it is possible to construct $\mathcal{N}(\bar{G})$, and then invoke the result of Hagos $[6]$ in the induction step. Hagos proved that the pair $(P(G ; \lambda), P(\bar{G} ; \lambda))$ can be reconstructed from the collection $\{(P(G-u ; \lambda), P(\bar{G}-$ $u ; \lambda)) ; u \in V G\}$. We skip the details of this argument. The proof presented here counts many other invariants. It is likely that the deck of pairs of polynomials considered by Hagos contains enough information for counting hamiltonian cycles and spanning trees.

Now we count the subgraphs with a given number of components, and a given number of edges in each component, and use it to compute the rank polynomial.

Let $\mathcal{G}\left(p, l,\left(p_{i}, q_{i}\right)_{i=1}^{l}\right)$ be the family of graphs with $p$ vertices and $l$ components, such that the $i$ 'th component has $p_{i}$ vertices and $q_{i}$ edges for $i \in[1, l]$. So, $\sum_{i} p_{i}=p$. We also assume that $p_{i} \geq p_{j}$ whenever $i<j$. By extending the notation $\left[\begin{array}{c}G \\ F\end{array}\right]$ defined earlier, we denote by $\left[\begin{array}{c}G \\ \mathcal{G}\left(p, l,\left(p_{i}, q_{i}\right)_{i=1}^{l}\right)\end{array}\right]$ the number of subgraphs of $G$ that belong to the family $\mathcal{G}\left(p, l,\left(p_{i}, q_{i}\right)_{i=1}^{l}\right)$.

Lemma 3.13. The number of connected spanning subgraphs of $G$ with $k$ edges, that is, $\left[\begin{array}{c}G \\ \mathcal{G}(v(G), 1,(v(G), k))\end{array}\right]$, is reconstructible from $\mathcal{N}(G)$ for all $k$.

Proof. When $k<v(G)-1,\left[\begin{array}{c}G \\ \mathcal{G}(v(G), 1,(v(G), k))\end{array}\right]$ is 0 . When $k \in[v(G)-1, e(G)]$, we prove the result by induction on $k$. The base case $k=v(G)-1$, which corresponds to the number of spanning trees, was proved in Theorem 3.12. Let the claim be true for all $k \in[v(G)-1, q-1]$. To prove the claim for $k=q$, define $A \equiv\left(a_{i}\right)_{i=1}^{q}$ such that $a_{i}=2$ 
for all $i \leq q$. We can write

$$
\operatorname{con}(A \rightarrow G)=\sum_{i=n-1}^{q} i ! S(q, i)\left[\begin{array}{c}
G \\
\mathcal{G}(v(G), 1,(v(G), i))
\end{array}\right]
$$

In Theorem 3.12, $\operatorname{con}(A \rightarrow G)$ was shown to be reconstructible from $\mathcal{N}(G)$. By the induction hypothesis, all terms on the RHS, except $[\mathcal{G}(v(G), 1,(v(G), q))]$, are known. Solving Equation (27) for $[\mathcal{G}(v(G), 1,(v(G), q))]$ completes the induction step and the proof.

Lemma 3.14. $\left[\begin{array}{c}G \\ \mathcal{G}\left(v(G), l,\left(n_{i}, m_{i}\right)_{i=1}^{l}\right)\end{array}\right]$, where $\sum_{i=1}^{l} n_{i}=v(G)$ and $m_{i}>0$ for all $i$, is reconstructible from $\mathcal{N}(G)$.

Proof. Let $A \equiv\left(A_{i}\right)_{i=1}^{l}$, where $A_{i} \equiv\left(a_{i j}\right)_{j=1}^{m_{i}} ; a_{i j}=2 \forall i, j$, and $B \equiv\left(n_{i}\right)_{i=1}^{l}$. By Theorem 3.12, $T_{v(G)}(A, B \rightarrow G)$ is $\mathcal{N}$-matrix reconstructible. We first express $T_{v(G)}(A, B \rightarrow G)$ in terms of the subgraphs to be counted, and then count the subgraphs by induction.

$$
\begin{aligned}
& T_{v(G)}(A, B \rightarrow G)=\sum_{\substack{\left(X_{1}, X_{2}, \ldots, X_{X}\right)\left| \\
\cup_{j}^{l} X_{j}=V=1\\
\right| X_{j} \mid=n_{j} \forall j}} \prod_{i=1}^{l} \operatorname{con}\left(A_{i} \rightarrow G_{X_{i}}\right) \\
& =\sum_{\left(X_{1}, X_{2}, \ldots, X_{l}\right) \mid} \prod_{i=1}^{l}\left(\sum_{q_{i}=n_{i}-1}^{m_{i}} q_{i} ! S\left(m_{i}, q_{i}\right)\left[\begin{array}{c}
G_{X_{i}} \\
\mathcal{G}\left(n_{i}, 1,\left(n_{i}, q_{i}\right)\right)
\end{array}\right]\right) \\
& \begin{array}{c}
\cup_{j=1}^{l} X_{j}=V(G) \\
\mid X_{j}=n_{j} \forall j
\end{array} \\
& =\sum_{\substack{\left(X_{1}, X_{2}, \ldots, X_{l}\right)\left| \\
\cup_{j=1}^{l} X_{j}=V(G)\\
\right| X_{j} \mid=n_{j} \forall j}} \sum_{\substack{\left(q_{1}, q_{2}, \ldots, \ldots q_{l}\right) \mid \\
n_{j}-1 \leq q_{j} \leq m_{j} \forall j}} \prod_{i=1}^{l}\left(q_{i} ! S\left(m_{i}, q_{i}\right)\left[\begin{array}{c}
G_{X_{i}} \\
\mathcal{G}\left(n_{i}, 1,\left(n_{i}, q_{i}\right)\right)
\end{array}\right]\right) \\
& =\sum_{\substack{\left(q_{1}, q_{2}, \ldots, q_{l}\right) \mid \\
n_{j}-1 \leq q_{j} \leq m_{j} \forall j}} \sum_{\substack{\left(X_{1}, X_{2}, \ldots, X_{l}\right)\left| \\
\cup_{j=1}^{l} X_{j}=V(G)\\
\right| X_{j} \mid=n_{j} \forall j}} \prod_{i=1}^{l}\left(q_{i} ! S\left(m_{i}, q_{i}\right)\left[\begin{array}{c}
G_{X_{i}} \\
\mathcal{G}\left(n_{i}, 1,\left(n_{i}, q_{i}\right)\right)
\end{array}\right]\right) \\
& =\sum_{\substack{\left(q_{1}, q_{2}, \ldots, q_{l}\right) \mid \\
n_{j}-1 \leq q_{j} \leq m_{j} \forall j}}\left(\prod_{i=1}^{l} q_{i} ! S\left(m_{i}, q_{i}\right)\right)\left(\sum_{\substack{\left(X_{1}, X_{2}, \ldots, X_{l}\right)\left| \\
\cup_{j}^{l} X_{j}=V(G) \\
j X_{j}\right|=n_{j} \forall j}}^{l}\left[\begin{array}{c}
G_{X_{i}} \\
\mathcal{G}\left(n_{i}, 1,\left(n_{i}, q_{i}\right)\right)
\end{array}\right]\right)
\end{aligned}
$$


The sequence $\left(n_{i}, q_{i}\right)_{i=1}^{l}$ may be written as $\left(n_{i}^{\prime}, q_{i}^{\prime}\right)^{\mu_{i}} ; i=1$ to $r$, which denotes that the pair $\left(n_{i}^{\prime}, q_{i}^{\prime}\right)$ appears $\mu_{i}$ times in the sequence $\left(n_{i}, q_{i}\right)_{i=1}^{l}$, the pairs $\left(n_{i}^{\prime}, q_{i}^{\prime}\right)$ are all distinct for $i=1$ to $r$, and that $\sum_{i=1}^{r} \mu_{i}=l$. Then each subgraph of $G$ that belongs to the family $\mathcal{G}\left(v(G), l,\left(n_{i}, q_{i}\right)_{i=1}^{l}\right)$ is counted $\prod_{i=1}^{r} \mu_{i}$ ! times in the inner summation. Therefore,

$$
T_{v(G)}(A, B \rightarrow G)=\sum_{\substack{\left(q_{1}, q_{2}, \ldots, q_{l}\right) \mid \\
n_{j}-1 \leq q_{j} \leq m_{j} \forall j}}\left(\prod_{i=1}^{l} q_{i} ! S\left(m_{i}, q_{i}\right)\right)\left(\prod_{i=1}^{r} \mu_{i} !\right)\left[\begin{array}{c}
G \\
\mathcal{G}\left(v(G), l,\left(n_{i}, q_{i}\right)_{i=1}^{l}\right)
\end{array}\right]
$$

The LHS of Equation (29) is known by Theorem 3.12. Now we prove the claim by induction on $\sum_{i} m_{i}$. The base case of induction corresponds to the case in which each component in the subgraphs being counted has minimum number of edges, that is, $m_{i}=$ $n_{i}-1$ for all $i \leq l$. In this case, there is only one term on the RHS of Equation (29), and it contains the unknown $\left[\mathcal{G}\left(v(G), l,\left(n_{i}, n_{i}-1\right)_{i=1}^{l}\right)\right]$, which can be solved for. Suppose the claim is true for $\sum_{i} m_{i}<m$. Now let $\sum_{i} m_{i}=m$. In this case, as in Lemma 3.13, there is only one unknown $\left[\mathcal{G}\left(v(G), l,\left(n_{i}, m_{i}\right)_{i=1}^{l}\right)\right]$ on the RHS of Equation (29). All other terms on the RHS are known by the induction hypothesis. We can compute the unknown term to obtain the desired result. This completes the induction step and the proof.

Theorem 3.15. The rank polynomial $R(G ; x, y)$ is reconstructible from $\mathcal{N}(G)$.

Proof. Lemma 3.14 can be applied to all induced subgraphs of $G$. So, we can count the number of subgraphs with $v$ vertices (none of which isolated), $e$ edges and $l$ components for all $v \leq v(G), e \leq e(G)$ and $l \geq 1$. Therefore, $\rho_{r s}$ in the expression for the rank polynomial are known.

\section{Computing $P(G ; \lambda)$ from $\mathcal{P} \mathcal{D}(G)$}

In this section, we consider the problem of computing the characteristic polynomial of a graph from its complete polynomial deck. We prove that elementary spanning subgraphs of each type other than hamiltonian cycles can be counted from the complete polynomial deck of a graph, thus proving that the characteristic polynomial of a non-hamiltonian graph is reconstructible from its complete polynomial deck.

Here we apply the idea of Kocay's Lemma to the coefficients of the characteristic polynomials.

Let $A \equiv\left(a_{i}\right)_{i=1}^{k}$ be a non-increasing sequence in $[2, v(G)]$. In this section, we define the notation $p\left(A \rightarrow G_{X}\right)$ and $c\left(A \rightarrow G_{X}\right)$ differently. 


$$
\begin{aligned}
p\left(A \rightarrow G_{X}\right)= & \prod_{j=1}^{k}(-1)^{a_{j}} c_{a_{j}}\left(G_{X}\right) \\
= & \prod_{j=1}^{k}\left(\sum_{F \subseteq G_{X}, F \in L_{a_{j}}}(-1)^{r(F)} 2^{s(F)}\right) \\
= & \sum_{(\forall j \in[1, k])\left(F_{1}, F_{2}, \ldots, F_{X}\right) \mid}\left((-1)^{\sum_{j=1}^{k} r\left(F_{j}\right)} 2^{\sum_{j=1}^{k} s\left(F_{j}\right)}\right) \\
= & \sum_{Y \subseteq X} c\left(A \rightarrow G_{Y}\right)
\end{aligned}
$$

where

$$
c\left(A \rightarrow G_{Y}\right)=\sum_{\substack{\left(F_{1}, F_{2}, \ldots, F_{k}\right) \mid \\(\forall j \in[1, k])\left(F_{j} \subseteq G_{Y}, F_{j} \in L_{a_{j}}\right) \\ \bigcup_{j=1}^{k}\left(V F_{j}\right)=Y}}\left((-1)^{\sum_{j=1}^{k} r\left(F_{j}\right)} 2^{\sum_{j=1}^{k} s\left(F_{j}\right)}\right)
$$

Thus we have grouped together tuples of elementary subgraphs in groups that span each subset of $X$.

Lemma 4.1. If $A$ is a sequence defined over $[2, v(G)-1]$ then $c(A \rightarrow G)$ is reconstructible from the complete polynomial deck of $G$.

Proof. As in the proof of Lemma 3.5, $p\left(A \rightarrow G_{X}\right)$ can be computed for each induced subgraph $G_{X}$. By Möbius inversion of Equation (30), we write

$$
c\left(A \rightarrow G_{X}\right)=\sum_{Y \subseteq X}(-1)^{|X \backslash Y|} p\left(A \rightarrow G_{Y}\right)
$$

But we cannot compute the RHS of Equation (32) because, in general, for $X \subsetneq V G$, we do not know which polynomials in $\mathcal{P} \mathcal{D}(G)$ correspond to the induced subgraphs of $G_{X}$. But this is not a problem if $X=V G$. We can write

$$
c(A \rightarrow G)=\sum_{Y \subseteq V G}(-1)^{|V G \backslash Y|} p\left(A \rightarrow G_{Y}\right)
$$

Now the RHS, and hence $c(A \rightarrow G)$, can be computed.

Remark. Note that we would not be able to compute $p(A \rightarrow G)$ if we defined $A$ in $[2, v(G)]$, because we do not know $c_{v(G)}(G)$.

Let $\lambda(m, p) \equiv\left(x_{1}, x_{2}, \ldots, x_{p}\right)$ denote a partition of $m$. We assume that $x_{1} \geq x_{2} \ldots \geq$ $x_{p}$. We write $\lambda(m)$ when the number of parts $p$ is not relevant. Also, we just write $\lambda$ instead of $\lambda(m, p)$ when $m$ and $p$ are either understood from the context or not relevant. 
Another partition $\lambda^{\prime}(m, p+1)$ can be obtained from $\lambda(m, p)$ by replacing an $x_{i}$ by $y$ and $z$ such that $y+z=x_{i}$, and ordering the numbers in a non-increasing order. Any partition that is obtained from $\lambda(m, p)$ by a sequence of such operations is called a refinement of $\lambda(m, p)$. Also, $\lambda(m, p)$ is a trivial refinement of itself. If $\lambda^{\prime}(m, q)$ is a refinement of $\lambda(m, p)$, then we denote it by $\lambda^{\prime}(m, q) \preceq \lambda(m, p)$. The relation $\preceq$ between partitions is a partial order.

Now consider partitions in which the smallest part $x_{p}$ is at least 2. Associated with each such partition $\lambda(m, p)$, there is a unique elementary graph $F_{\lambda} \in L_{m}$, whose $i$ 'th component is a cycle of length $x_{i}$, or an edge if $x_{i}=2$. If the sequence $A \equiv\left(a_{i}\right)_{i=1}^{k}$ is defined such that $\lambda \equiv\left(a_{1}, a_{2}, \ldots, a_{k}\right)$ is a non-trivial partition of $v(G)$, then we denote $c(A \rightarrow G)$ by $c(\lambda \rightarrow G)$.

Lemma 4.2. Let $\lambda \equiv\left(a_{1}, a_{2}, \ldots, a_{k}\right)$ be a non-trivial partition of $n=v(G)$. Then,

$$
c(\lambda \rightarrow G)=\sum_{\lambda^{\prime} \preceq \lambda} c\left(\lambda \rightarrow F_{\lambda^{\prime}}\right)\left[\begin{array}{c}
G \\
F_{\lambda^{\prime}}
\end{array}\right]
$$

Proof. From the definition of $c(A \rightarrow G)$, we write

$$
\begin{aligned}
c(\lambda \rightarrow G)= & \sum_{\substack{\left(F_{1}, F_{2}, \ldots, F_{k}\right) \mid \\
(\forall j \in[1, k])\left(F_{j} \subseteq G, F_{j} \in L_{a_{j}}\right) \\
\bigcup_{j=1}^{k}\left(V F_{j}\right)=V G}}\left((-1)^{\sum_{j=1}^{k} r\left(F_{j}\right)} 2^{\sum_{j=1}^{k} s\left(F_{j}\right)}\right) \\
= & \sum_{F \subseteq G, F \in L_{n}} \sum_{\substack{\left(F_{1}, F_{2}, \ldots, F_{k}\right) \mid \\
(\forall j \in[1, k])\left(F_{j} \subseteq F, F_{j} \in L_{a_{j}}\right) \\
\bigcup_{j=1}^{k} F_{j}=F}}\left((-1)^{\sum_{j=1}^{k} r\left(F_{j}\right)} 2^{\sum_{j=1}^{k} s\left(F_{j}\right)}\right) \\
= & \sum_{F \subseteq G, F \in L_{n}} c(\lambda \rightarrow F) \\
= & \sum_{\lambda^{\prime} \preceq \lambda} c\left(\lambda \rightarrow F_{\lambda^{\prime}}\right)\left[\begin{array}{c}
G \\
F_{\lambda^{\prime}}
\end{array}\right]
\end{aligned}
$$

The last step may be explained as follows: if $F$ is a disjoint union of elementary graphs $F_{j} \in L_{a_{j}} ; j \in[1, k]$, where $v(F)=v(G)=n$, then $F$ is isomorphic to an elementary graph $F_{\lambda^{\prime}}$ for some refinement $\lambda^{\prime}$ of $\lambda$. Trivially, if each $F_{j}$ is the cycle $C_{a_{j}}$ then $F=F_{\lambda}$. We then group the terms $c(\lambda \rightarrow F)$ by the isomorphism type of $F$.

Lemma 4.3. If $F$ is an elementary graph on $n=v(G)$ vertices, other than the cycle, then $\left[\begin{array}{c}G \\ F\end{array}\right]$ is reconstructible from $\mathcal{P} \mathcal{D}(G)$.

Proof. Since $F$ is not a cycle, it is isomorphic to $F_{\lambda_{0}}$ for a unique non-trivial partition $\lambda_{0}$ of $v(G)$. From Equation (34) we write

$$
\left[\begin{array}{c}
G \\
F_{\lambda_{0}}
\end{array}\right]=\frac{1}{c\left(\lambda_{0} \rightarrow F_{\lambda_{0}}\right)}\left(c\left(\lambda_{0} \rightarrow G\right)-\sum_{\lambda \prec \lambda_{0}} c\left(\lambda_{0} \rightarrow F_{\lambda}\right)\left[\begin{array}{c}
G \\
F_{\lambda}
\end{array}\right]\right)
$$


Now we expand $\left[\begin{array}{c}G \\ F_{\lambda}\end{array}\right]$ on the RHS of the above equation by repeated application of the same equation, and obtain the following solution.

$$
\left[\begin{array}{c}
G \\
F_{\lambda_{0}}
\end{array}\right]=\sum_{\lambda_{q} \prec \lambda_{q-1} \prec \ldots \prec \lambda_{0}} \frac{(-1)^{q} c\left(\lambda_{q} \rightarrow G\right) \prod_{i=0}^{q-1} c\left(\lambda_{i} \rightarrow F_{\lambda_{i+1}}\right)}{\prod_{i=0}^{q} c\left(\lambda_{i} \rightarrow F_{\lambda_{i}}\right)}
$$

where the summation is over all chains $\lambda_{q} \prec \lambda_{q-1} \prec \ldots \prec \lambda_{0} ; q \geq 0$, and an empty product is 1 . There are finitely many terms in the above summation since there are finitely many refinements of $\lambda_{0}$. Since $\lambda_{0}$ is a non-trivial partition of $n$, (that is, $x_{i}<v(G) \forall i$ ), by Lemma 4.1, $c(\lambda \rightarrow G)$ is reconstructible for each $\lambda \preceq \lambda_{0}$. Also, for each $\lambda \preceq \lambda_{0}$, $c\left(\lambda \rightarrow F_{\lambda}\right)$ is non-zero. (Here we would like to repeat that we have considered only those partitions in which the smallest part is at least 2.) Thus the RHS can be computed.

The main theorem in this section now follows from the above lemmas.

Theorem 4.4. If $F$ is an elementary graph on $v(G)$ vertices, other than a cycle, then $\left[\begin{array}{l}G \\ F\end{array}\right]$ is reconstructible from $\mathcal{P} \mathcal{D}(G)$. Therefore, if there is a vertex of degree 1 in $G$, then the characteristic polynomial of $G$ is reconstructible from its complete polynomial deck.

Proof. The degree sequence of a graph is reconstructed from its complete polynomial deck as follows. Consider the polynomials of degree $v(G)-1$ in $\mathcal{P} \mathcal{D}(G)$. They are the characteristic polynomials of the vertex deleted subgraphs $G-u$ of $G$ for $u \in V G$. Since $c_{2}(G)$ and $c_{2}(G-u)$ count the number of edges of $G$ and $G-u$, respectively, we know the degree of $u$ in $G$ for each $u \in V G$. Thus the premise of Theorem 4.4 is recognised from $\mathcal{P D}(G)$. The coefficients $c_{i}(G) ; i<v(G)$ can be computed using Lemma 1.7. Since there are no hamiltonian cycles in $G$, Lemma 4.3 implies that the constant term in the characteristic polynomial of $G$ can be calculated.

Remark. Whenever non-hamiltonicity of a graph is recognised from its complete polynomial deck, its characteristic polynomial can be computed as well.

\section{Whitney's Theorem}

In Section 1, it was stated that the computation of the chromatic polynomial of a graph requires only non-separable induced subgraphs of the graph. Whitney's proof of this fact was based on his theorem that separable subgraphs can be counted from the counts of non-separable subgraphs. Let $n_{t}(G)$ denote the number of subgraphs of $G$ of type $t$, where 'type' of a graph is determined by the number of blocks of each isomorphism type. (Thus the graph type is not the isomorphism type in general, since non-isomorphic graphs having the same collection of blocks have the same graph type.) He proved, (stated in the terminology of [2]), that there is a polynomial $\phi_{t}$, independent of $G$, such that

$$
n_{t}(G)=\phi_{t}\left(n_{\sigma}(G), n_{\rho}(G), \ldots\right)
$$

THE EleCtronic Journal of COMBinatorics 12 (2005), \#R63 
where $\sigma, \rho, \ldots$ are non-separable types with not more edges than $t$. Here we prove Whitney's result using Kocay's Lemma. Our presentation explicitly describes the polynomial in Whitney's theorem.

Let $S_{0}=\left\{F_{1}, F_{2}, \ldots, F_{k}\right\}$ be a family of non-separable graphs, some of them possibly isomorphic. Thus $S_{0}$ represents a 'graph type'. Extending the notation introduced earlier, we write $\left[\begin{array}{c}G \\ S_{0}\end{array}\right]$ to denote the number of subgraphs of $G$ of type $S_{0}$. We define a partial order $\preceq$ on graph types as follows. Let $S$ be a graph type. We say that $S \preceq S_{0}$ if $c\left(S_{0}, X\right)$ is non-zero for some graph $X$ of type $S$. It is easily seen that $c\left(S_{0}, X\right)$ depends only on the type $S$ of $X$, not on a particular choice of $X$. So, we write it as $c\left(S_{0}, S\right)$. For any graph $G$, by Kocay's Lemma 1.3,

$$
p\left(S_{0}\right)=\prod_{i=1}^{k}\left[\begin{array}{c}
G \\
F_{i}
\end{array}\right]=\sum_{X} c\left(S_{0}, X\right)\left[\begin{array}{c}
G \\
X
\end{array}\right]
$$

Terms on the RHS can be grouped together according to the types of $X$, so we can write

$$
\begin{aligned}
p\left(S_{0}\right) & =\sum_{S \preceq S_{0}} c\left(S_{0}, S\right)\left[\begin{array}{c}
G \\
S
\end{array}\right] \\
& =c\left(S_{0}, S_{0}\right)\left[\begin{array}{c}
G \\
S_{0}
\end{array}\right]+\sum_{S \prec S_{0}} c\left(S_{0}, S\right)\left[\begin{array}{c}
G \\
S
\end{array}\right]
\end{aligned}
$$

Therefore,

$$
\left[\begin{array}{c}
G \\
S_{0}
\end{array}\right]=\frac{1}{c\left(S_{0}, S_{0}\right)}\left(p\left(S_{0}\right)-\sum_{S \prec S_{0}} c\left(S_{0}, S\right)\left[\begin{array}{c}
G \\
S
\end{array}\right]\right)
$$

Now we repeatedly apply the same equation to $\left[\begin{array}{c}G \\ S\end{array}\right]$ on the RHS, as we did in Equation (37). We thus get the polynomial of Whitney's theorem.

\section{Theorem 5.1.}

$$
\left[\begin{array}{c}
G \\
S_{0}
\end{array}\right]=\sum_{S_{q} \prec S_{q-1} \prec \ldots \prec S_{0}} \frac{(-1)^{q} p\left(S_{q}\right) \prod_{i=0}^{q-1} c\left(S_{i}, S_{i+1}\right)}{\prod_{i=0}^{q} c\left(S_{i}, S_{i}\right)}
$$

where the summation is over all chains $S_{q} \prec S_{q-1} \prec \ldots \prec S_{0} ; q \geq 0$, and an empty product is 1 .

There are finitely many terms in the summation in Equation (42) because each $S_{q}$ has fewer blocks in it than $S_{q-1}$. While some other known proofs of this theorem are based on not very different ideas, (for example, see [1]), the above explicit formulation of the polynomial seems new. It allows us to argue about the reconstructibility of the characteristic polynomial more directly than in other standard proofs. 
Corollary 5.2. The characteristic polynomial of a graph is reconstructible from its vertex deck.

Proof. Let $G$ be the graph under consideration. Its elementary spanning subgraphs other than the hamiltonian cycles are counted as in the standard proof. Let $n=v(G)$, and let $S_{0}$ be the type of an $n$-vertex elementary graph $H$ other than $C_{n}$. Any block in a type $S \prec S_{0}$ has fewer than $n$ vertices, and $H$ is the only graph of type $S_{0}$ that has $n$ vertices.

So $\left[\begin{array}{l}G \\ H\end{array}\right]$ can be counted using Whitney's Theorem 5.1 and Kelly's Lemma 1.2.

To count hamiltonian cycles, we set $S_{0}=\left\{n K_{2}\right\}$, that is, a graph type consisting of $n$ blocks, each one of them a $K_{2}$. Since no subgraph of $G$ has $n$ blocks isomorphic to $K_{2}$, $\left[\begin{array}{c}G \\ S_{0}\end{array}\right]=0$. On the RHS of Equation (42), there is precisely one term that contributes hamiltonian cycles. That is, $S_{1}=\left\{C_{n}\right\} \prec S_{0}$ is the unique chain that contributes $\operatorname{ham}(G)$, implying that the terms containing $\operatorname{ham}(G)$ cannot cancel out. All other blocks that appear in Equation (42) have fewer than $n$ vertices, so can be counted using Kelly's Lemma 1.2. Therefore, $\operatorname{ham}(G)$ is reconstructible. The reconstructibility of $P(G ; \lambda)$ now follows from Lemma 1.6.

Remark. In the standard proof of the reconstructibility of the characteristic polynomial, one applies Kocay's Lemma directly. As a result one has to proceed step by step, counting spanning trees, then spanning unicyclic subgraphs, etc., as we did in Corollary 3.9. These intermediate steps are skipped by the direct application of Whitney's theorem.

\section{Problems and discussion}

Expressing $c_{n}(G)$ or $\psi_{n}(G)$, where $n=v(G)$, as polynomials in $c_{j}(G-S)$ or $\psi_{j}(G-S)$; $S \subsetneq V G$, would be of interest. Alternatively, we would like to construct a generalisation of the characteristic polynomial which can be computed more naturally from the poset of induced subgraphs, and from which the characteristic polynomial can be easily computed. Such a goal is motivated on the one hand by the proofs in Section 3, and, on the other hand, by similar generalisations of the chromatic polynomial, viz, Stanley's chromatic symmetric function, (see [11] \& [13]), and another recent two variable generalisation of the chromatic polynomial [4]. Both these generalisations are closely related to the lattice of connected partitions of $V G$, (see [11] for definitions). A relationship between the poset of induced subgraphs defined in this paper and the lattice of connected partitions of the vertex set defined by Stanley could possibly be established using Kocay's Lemma. Such a result would be useful in understanding exact relationship between different expansions (and reconstructibility) of several important invariants in a unified way.

The reconstruction of the number of hamiltonian cycles is difficult and indirect in the proofs we have presented here, and in the original proof by Tutte as well. A reason for this difficulty is seen in Whitney's theorem. Observe that for an $n$-vertex graph $G$, the polynomial of Whitney's theorem contains a term in $\operatorname{ham}(G)$ only if the type $S_{0}$ contains $n$ copies of $K_{2}$, or a $C_{n}$, and possibly other types of blocks. As a result, in Corollary 5.2 
we had to count all possible blocks with at most $n$ edges. But can we count the number of hamiltonian cycles from the $\mathcal{N}$-matrix at least as clearly as in Corollary 5.2? Towards this goal, we would like to understand the relationship between the structure of the edge labelled poset for separable graphs and that for blocks, and prove a generalisation of Whitney's theorem.

The crucial difference between the proofs in Section 3 and Section 4 is in Lemmas 3.5 and 4.1. In Lemma 4.1, the use of Möbius inversion was limited to the computation of $c(A \rightarrow G)$ because we did not know the partial order on the induced subgraphs. This suggests that a general 'expansion' for the number of hamiltonian cycles would probably involve a summation over chains in $\mathcal{E} \mathcal{L P}(G)$. Therefore, counting hamiltonian cycles from $\mathcal{P} \mathcal{D}(G)$ and the original problem of Gutman and Cvetkovic seem difficult. This is probably why many known results on the reconstruction of the characteristic polynomial of a graph from its characteristic polynomial deck assume the graph to contain several pendant vertices.

We propose the following generalisation of reconstruction for studying questions similar to the one posed by Gutman and Cvetković. Suppose $f$ is a graph invariant, and we are interested in reconstructing $G$ or partial invariants of $G$ from the $\operatorname{deck} \mathcal{D}(G ; f)=\{f(G-$ $u) ; u \in V G\}$. A new collection $\mathcal{D} !(G ; f)$ is recursively defined as $\{(f(G-u), \mathcal{D} !(G ; f-$ $u)$ ) $u \in V G\}$. We then define an equivalence relation $\sim$ on graphs such that $H_{1} \sim H_{2}$ if $\left(f\left(H_{1}\right), \mathcal{D} !\left(H_{1}\right)\right)=\left(f\left(H_{2}\right), \mathcal{D} !\left(H_{2}\right)\right)$. This relation gives an incidence matrix (or an edge labelled poset) on the types of induced subgraphs of $G$, where 'type' refers to an equivalence class under the relation $\sim$ defined above. It can be shown that for many invariants $f$, Ulam's conjecture is true if and only if all graphs $G$ on more than 2 vertices are reconstructible from $\mathcal{D} !(G ; f)$. One example of such an invariant is: $f(G)=1$ if $G$ has a vertex of degree 1 , and $f(G)=0$ otherwise. Another example is $f(G)=P(G ; \lambda)$. The proof of this is similar to that of Proposition 2.7: the base case follows from the fact that any three vertex graph $G$ is completely determined by $\mathcal{D} !(G ; f)$ for the above invariants. The problem of reconstructing $G$ from the deck $\mathcal{D} !(G ; f)$ is similar to the generalisation of the reconstruction problem suggested by Tutte, (Notes on pp. 123-124 in [15]). We are not really interested in the question of computing $f(G)$ from $\mathcal{D}(G ; f)$. Rather we ask the question - what are the incomplete invariants $f$, (that is, the invariants that do not determine a graph completely,) and classes of graphs $G$, for which $\mathcal{D} !(G ; f)$ could be constructed from $\mathcal{D}(G ; f)$ ? If we could construct $\mathcal{D} !(G ; f)$ from $\mathcal{D}(G ; f)$, then we could also prove all the results of Section 3. We would like to investigate this question when $f(G)=P(G ; \lambda)$, and when $f(G)=(P(G ; \lambda), P(\bar{G} ; \lambda))$ - the invariant which was considered by Hagos [6].

\section{Acknowledgements}

I take this opportunity to thank Allan Wilson Centre for the support and encouragement. I would also like to thank the referee for several useful suggestions for improving the presentation. 


\section{References}

[1] N. Biggs. On cluster expansions in graph theory and physics. Quart. J. Math. (Oxford), 29:159-173, 1970.

[2] N. Biggs. Algebraic Graph Theory. Cambridge University Press, 1993.

[3] A. Bondy. A graph reconstructor's manual. Surveys in Combinatorics, London Math. Soc. Lecture Note Ser. 166, pages 221-252, 1991.

[4] K. Dohmen, A. Pönitz, and P. Tittmann. A new two variable generalisation of the chromatic polynomial. Discrete Mathematics and Theoretical Computer Science, 6:069-090, 2003.

[5] I. Gutman and D. M. Cvetković. The reconstruction problem for the characteristic polynomial of graphs. Publ. Electrotehn, Fac. Ser. Fiz. No.488-541, pages 45-48, 1975.

[6] Elias M. Hagos. The characteristic polynomial of a graph is reconstructible from the characteristic polynomials of its vertex-deleted subgraphs and their complements. Electronic Journal of Combinatorics, 7:\#R12, 2000.

[7] P. J. Kelly. A congruence theorem for trees. Pacific J. Math., 7:961-968, 1957.

[8] W. L. Kocay. On reconstructing spanning subgraphs. Ars Combin., 11:301-313, 1981.

[9] A. J. Schwenk. On the eigenvalues of a graph. In L. W. Beineke and R. J. Wilson, editors, Selected Topics in Graph Theory, pages 307-336. Academic Press, New York, 1979.

[10] I. Sciriha. Polynomial reconstruction and terminal vertices. Linear Algebra and its Applications, 356:145-156, 2002.

[11] R. P. Stanley. A symmetric function generalisation of the chromatic polynomial of a graph. Advances in Mathematics, 111:166-194, 1995.

[12] R. P. Stanley. Enumerative Combinatorics, vol 1. Cambridge University Press, 1997.

[13] R. P. Stanley. Enumerative Combinatorics, vol 2. Cambridge University Press, 1999.

[14] W. T. Tutte. All the king's horses. In J.A.Bondy and U.S.R.Murthy, editors, Graph Theory and Related Topics, (Proceedings of the conference held in honour of W. T. Tutte on the occasion of his 60th birthday, Waterloo 1977), pages 15-33. Academic Press, New York, 1979.

[15] W. T. Tutte. Graph Theory, Encyclopedia of Mathematics and its Applications, vol 21. Addison-Wesley Publishing Co., Reading, Mass., 1984.

[16] S. Ulam. A Collection of Mathematical Problems. Wiley (Interscience), New York, 1960.

[17] H. Whitney. A logical expansion in mathematics. Bull. Amer. Math. Soc., 38:572579, 1932. 